\title{
20. Organisationale Struktur des freiwilligen Engagements und Verbesserungsmöglichkeiten der Rahmenbedingungen
}

\author{
Julia Simonson \& Claudia Vogel
}

\section{Kernaussagen}

Freiwilliges Engagement findet anteilig am häufigsten in Vereinen und Verbänden statt. Über die Hälfte der Engagierten ist im Rahmen dieser Organisationsform freiwillig tätig, gefolgt von individuell organisierten Gruppen, Kirchen und religiösen Vereinigungen, anderen überwiegend formal organisierten Einrichtungen sowie kommunalen oder staatlichen Einrichtungen. Freiwillig engagierte Frauen sind zu einem geringeren Anteil in Vereinen und Verbänden tätig als freiwillig engagierte Männer und zu einem höheren Anteil in Kirchen oder religiösen Vereinigungen.

Individuell organisiertes Engagement gewinnt an Bedeutung. Der Anteil Engagierter in individuell organisierten Gruppen hat von 11,0 Prozent im Jahr 1999 auf 16,0 Prozent im Jahr 2014 zugenommen. Hingegen hat der Anteil Engagierter in Vereinen und Verbänden im Zeitvergleich abgenommen.

Organisationen stellen Ansprechpersonen bereit und ermöglichen Mitsprache. Knapp zwei Drittel aller Engagierten sind in einer Organisation freiwillig tätig, in der eine Ansprechpartnerin oder ein Ansprechpartner für die Ehrenamtlichen oder Freiwilligen vorhanden ist. In religiösen Vereinigungen und staatlichen oder kommunalen Einrichtungen gibt es solche Ansprechpersonen anteilig häufiger als in anderen Organisationformen. Mehr als drei Viertel aller Engagierten bewerten ihre Mitsprachemöglichkeiten als sehr gut beziehungsweise eher gut. Vor allem Engagierte in individuell organisierten Gruppen bewerten die Mitsprachemöglichkeiten als sehr gut beziehungsweise eher gut.

Engagierte sehen Verbesserungsbedarfe bei den Rahmenbedingungen freiwilligen Engagements. Verbesserungsbedarfe werden von den Engagierten seitens der Organisationen gesehen, für die sie freiwillig tätig sind, und in einem noch deutlicheren Ausmaß seitens des Staates und der Gesellschaft. Mehr als die Hälfte der Engagierten stimmt zu, dass mehr über Gelegenheiten zum ehrenamtlichen oder freiwilligen Engagement informiert und beraten werden soll.

Arbeitgeber unterstützen freiwillig engagierte Beschäftigte. Etwa ein Drittel der Engagierten, die auch einer abhängigen Beschäftigung nachgehen, wird von ihrem Arbeitgeber bei der Ausübung ihres Engagements unterstützt. Lediglich eine Minderheit von Engagierten (13,3 Prozent) dagegen wünscht sich Unterstützung für ihr freiwilliges Engagement vom Arbeitgeber, ohne eine solche zu erhalten. 


\subsection{Einleitung}

In der Regel engagieren sich Menschen in zivilgesellschaftlichen Organisationen. Dazu zählen zum Beispiel Vereine, kirchliche oder staatliche beziehungsweise kommunale Einrichtungen. Ein großer Teil des individuellen, freiwilligen Engagements ist gemeinsam mit dieser organisatorischen Infrastruktur der Zivilgesellschaft zu denken, denn die Organisationen schaffen Gelegenheiten, sich freiwillig zu engagieren und werben um Freiwillige (vgl. Alscher \& Priller 2011; Salamon \& Sokolowski 2003). Wir unterscheiden fünf Organisationsformen, von denen vier formal organisiert sind: 1) Vereine und Verbände, 2) Kirchen und religiöse Vereinigungen, 3) staatliche oder kommunale Einrichtungen, 4) andere überwiegend formal organisierte Einrichtungen sowie 5) individuell organisierte Gruppen. Letztere werden hauptsächlich durch die freiwillig Engagierten selbst getragen und organisiert und sie stehen zu großen Teilen für das Engagement von unten. Bereits seit den 1980er Jahren schließen sich Menschen vermehrt in individuell organisierten Gruppen wie Initiativen oder Selbsthilfegruppen zusammen (Sachße 2011). Dieses Engagement zeichnet sich nicht nur durch eine Vielfalt von unkonventionellen Projekten und Initiativen aus, sondern teils auch durch die Konkurrenz zu und der Kritik an den etablierten Wohlfahrtsverbänden, da in der Selbsthilfe die Interessen der Betroffenen vertreten sind, wie zum Beispiel in der Hospiz- und Palliativbewegung (Sachße 2011).

Ein Großteil des Engagements findet jedoch in formal organisierten Vereinigungen statt. Nach wie vor wird freiwilliges Engagement am häufigsten in Vereinen ausgeübt. Das liegt auch an der Vielzahl von Vereinen in Deutschland (mehr als 580.000 laut dezentralen Vereinsregistern; (Gensicke 2015; Krimmer \& Priemer 2013). Heute sind mehr Menschen Mitglied in mindestens einem Verein als noch 2009 und Vereinsmitglieder engagieren sich mit einer größeren Wahrscheinlichkeit freiwillig als Personen, die in keinem Verein Mitglied sind (siehe Kapitel 8). Die Rechtsform des Vereins als freiwilliger, auf gewisse Dau- er angelegter Zusammenschluss von mehreren Personen ist im Bürgerlichen Gesetzbuch (BGB $1, \$ \$ 21-89)$ geregelt. Wenn bürgerschaftliches Engagement in Vereinen stattfindet, dann laut Zimmer (2011) in der Regel in gemeinnützigen Vereinen, die ins Vereinsregister eingetragen und entsprechend am Zusatz e. V. (eingetragener Verein) zu erkennen sind. Sie sind nicht-wirtschaftliche Vereine und werden auch als Non-Profit-Organisationen bezeichnet. Neben einer Satzung braucht jeder Verein eine ausreichende Zahl an Mitgliedern und einen Vorstand, der sich aus Vorsitzenden und stellvertretenden Vorsitzenden sowie Schriftführerin beziehungsweise Schriftführer und Kassenwartin beziehungsweise Kassenwart zusammensetzt, also Personen, die sich freiwillig engagieren. Vereine und Verbände sind beides freiwillige Vereinigungen - die Verwendung dieser Begriffe ist in den Sozialwissenschaften ebenso wie in der Alltagssprache allerdings nicht trennscharf (vgl. Zimmer 2011; Heinze 2011). Zudem zeichnen sich Vereine durch eine große Vielfalt aus, auch Selbsthilfegruppen sind oft als Vereine organisiert, vielfach entwickeln sich Vereine in Richtung Service-, Dienstleistungs- und Lobbyeinrichtungen (Zimmer 2011: 462; Grunow 2011; Zeman 2012).

Zahlreiche Menschen engagieren sich auch in kirchlichen oder religiösen Vereinigungen. Das ehrenamtliche und freiwillige Engagement hat in den Kirchen eine lange Tradition (Coenen-Marx 2011). Dabei ist auch hier die Abgrenzung nicht trennscharf, da kirchliche Vereinigungen häufig die Rechtsform eines Vereins oder eines Verbandes nutzen. Auch der Staat sowie kommunale Einrichtungen bieten Gelegenheiten für freiwilliges Engagement. Eine traditionelle Form freiwilligen Engagements ist das kommunalpolitische Ehrenamt, also beispielsweise die ehrenamtliche Übernahme eines Bürgermeisteramtes. Kommunalpolitische Ehrenämter können auf unterschiedlichen Ebenen wahrgenommen werden, so zum Beispiel auf der Gemeinde-, Kreis- oder Bezirksebene (siehe auch für eine Übersicht Rei- 
ser 2011). In den letzten Jahren wurde allerdings verstärkt berichtet, es sei schwierig Kandidaten für ehrenamtliche Positionen in der Kommunalverwaltung zu finden (Reiser 2011: 298f.). Dies kann auf generell sinkende Mitgliederzahlen in Parteien und eine steigende Unzufriedenheit mit etablierten politischen Strukturen zurückzuführen sein. Möglicherweise hängt dies aber auch mit der geringeren Bereitschaft sich längerfristig an ein politisches Amt zu binden sowie der (aufgrund des hohen Zeitaufwands) schwierigen Vereinbarkeit mit Beruf und Familie zusammen (zu Hinderungsgründen siehe Kapitel 4).

Unterschiedliche zivilgesellschaftliche Akteure klagen über einen Mangel an Mitgliedern und insbesondere an Engagierten in ihren Organisationen (Alscher, Droß, Priller \& Schmeißer 2013). Die Daten des Freiwilligensurveys ermöglichen es zu überprüfen, inwieweit sich ein Trend zu einem Engagement außerhalb von formalen Organisationen abzeichnet und ob Engagement in individuell organisierten Gruppen an relativer Bedeutung zunimmt. Hier könnte das Wachstumspotenzial des freiwilligen Engagements liegen. Es kann davon ausgegangen werden, dass Engagement in individuell organisierten Gruppen andere Bevölkerungsgruppen anspricht als klassisches vereinsoder institutionengebundenes Engagement. Deshalb untersuchen wir, ob Frauen sich zu höheren Anteilen in individuell organisierten Gruppen engagieren als Männer, und welche Unterschiede sich zwischen Alters- und Bildungsgruppen bezüglich der Wahl der Organisationsform ihres Engagements zeigen. So könnten sich beispielsweise jüngere Menschen stärker von informellen Angeboten angesprochen fühlen als Ältere. Dieser Zusammenhang wird auch in der Forschung zur politischen Partizipation diskutiert, wobei davon ausgegangen wird, dass Jüngere stärker als Ältere zu alternativen Partizipations- und Protestformen neigen. Die Beteiligung Jüngerer an Unterschriftensammlungen, Demonstrationen und Bürgerinitiativen ist allerdings nicht höher als im Bevölkerungsdurchschnitt (siehe Kapitel 6). Diese Formen der Partizipation gehören mittlerweile $\mathrm{zu}$ den etablierten politischen Be- teiligungsformen aller Bürgerinnen und Bürger (Weßels 2013: 365). Zudem setzt individuell organisiertes Engagement möglicherweise höhere Anforderungen voraus, sodass Höhergebildete sich in dieser Form eher betätigen können.

Für zivilgesellschaftliche Akteure stellt sich die Frage, wie sie Personen für das Engagement in ihren Organisationen gewinnen und halten können (siehe Kapitel 4). Ein wichtiger Aspekt dabei ist, wie die Rahmenbedingungen für das Engagement verbessert werden können. Mit den Daten des Freiwilligensurveys lässt sich zeigen, was die Organisationen bisher für ihre Engagierten tun, zum Beispiel ob sie Ansprechpartnerinnen und -partner für die Engagierten vorsehen. Außerdem wird untersucht, wie Engagierte die Mitsprachemöglichkeiten in den Organisationen bewerten. Dies erlaubt Auskunft darüber, wie sich Engagierte in Entscheidungs- und Planungsprozesse eingebunden fühlen. Es hat sich gezeigt, dass hierfür insbesondere die Kooperation zwischen hauptamtlichen und ehrenamtlichen Kräften entscheidend ist (Schumacher 2015).

Darüber hinaus kann dargestellt werden, wo die Engagierten selbst Verbesserungsbedarfe sehen und wie die zivilgesellschaftlichen Organisationen und der Staat die Rahmenbedingungen für Engagement verbessern können: Eher durch Maßnahmen, die direkt auf die Projekte der Engagierten zielen, wie etwa die Bereitstellung von Ausstattungsmitteln und Information, oder durch Maßnahmen, die den Engagierten zugutekommen, zum Beispiel eine bessere Anerkennung oder Vergütung.

In dieser Debatte spielt die Anerkennungskultur eine wichtige Rolle, denn „Anerkennung ist eine der wichtigsten Formen der Förderung bürgerschaftlichen Engagements" (Enquete-Kommission ,Zukunft des Bürgerschaftlichen Engagements' 2002: 6). Die Enquete-Kommission hat empfohlen, dass Engagementpolitik die Entwicklung einer umfassenden Anerkennungskultur zum Ziel haben sollte, da diese unter anderem zur öffentlichen Sichtbarkeit bürgerschaftlichen Engagements beiträgt. So lassen sich verschiedene Formen der Anerkennung unterscheiden, 
zum Beispiel die immaterielle Anerkennung (Auszeichnungen, Ehrenmitgliedschaften etc.), die geldwerte Anerkennung (kostenlose Nutzung des öffentlichen Nahverkehrs, Freistellung oder Bildungsurlaub etc.), die monetäre Anerkennung (Vergütung, Aufwandsentschädigung oder Steuerbefreiung) und Anerkennung durch Qualifizierung (Enquete-Kommission, Zukunft des Bürgerschaftlichen Engagements' 2002: 127ff.). Es wird untersucht, bei welchen Anerkennungsformen die Engagierten am häufigsten Verbesserungsbedarf sehen und welche Gruppenunterschiede sich hierbei zeigen.

Darüber hinaus beeinflusst auch die Anerkennung außerhalb der Organisationen, ob und wie sich Menschen engagieren. Beispielsweise kann die Akzeptanz des freiwilligen Engagements im Berufsleben die Engagierten unterstützen, aber fehlende Akzeptanz das Engagement behindern. Zusätzlich wirken sich soziale Faktoren auf das Engagement aus. So engagieren sich Frauen nicht nur insgesamt anteilig seltener als Männer (siehe Kapitel 3), sondern auch seltener in Vereinen und Verbänden. Außerdem üben sie seltener eine Leitungs- oder Vorstandsfunktion aus (siehe Kapitel 11). Die Frage der Vereinbarkeit des Berufs nicht nur mit der Familie, sondern auch mit freiwilligem Engagement könnte hier ebenso eine Rolle spielen wie die Vereinbarkeit mit informellen Unterstützungsleistungen für Nachbarinnen und Nachbarn, Freundinnen und Freunde oder Bekannte. Berufstätige Frauen erfahren häufiger als berufstätige Männer eine Doppelbelastung durch Familie und Beruf, die möglicherweise eine freiwillige Tätigkeit oder private Unterstützungsleistungen in der Nachbarschaft erschwert, da damit eine Dreifachbelastung verbunden sein kann (Alscher 2010; Backes 2011). Es soll deshalb der Frage nachgegangen werden, ob von den Engagierten bezüglich der Vereinbarkeit von Beruf und Engagement Verbesserungsbedarfe gesehen werden, und ob berufstätige Frauen anteilig genauso häufig Unterstützung durch den Arbeitgeber für ihr Engagement erhalten wie berufstätige Männer.
In diesem Kapitel wird folgenden Forschungsfragen nachgegangen:

1. In welchen Organisationsformen engagieren sich Menschen freiwillig und lassen sich hierbei Unterschiede zwischen Bevölkerungsgruppen aufzeigen?

2. Zeichnet sich im Fünfzehn-Jahres-Zeitraum tatsächlich ein Trend $a b$, dass sich weniger Menschen in formalen Organisationen wie Vereinen und Verbänden und mehr Menschen außerhalb formaler Organisationen, etwa in Projekten oder selbstorganisierten Gruppen, freiwillig engagieren?

3. Sehen Organisationen Ansprechpartnerinnen und -partner für die freiwillig Engagierten vor und wie unterscheiden sich die Organisationsformen dabei?

4. Wie bewerten freiwillig Engagierte die Mitsprachemöglichkeiten in den Organisationen? Welche Unterschiede bestehen zwischen den verschiedenen Organisationsformen und Personengruppen?

5. Bei welchen Themen sehen die freiwillig Engagierten am häufigsten Verbesserungsbedarf auf Seiten der Organisationen und wie unterscheiden sich die Organisationsformen dabei?

6. Bei welchen Themen sehen die freiwillig Engagierten am häufigsten Verbesserungsbedarf auf Seiten des Staates und der Öffentlichkeit und wie unterscheiden sich die Organisationsformen dabei?

7. Schließlich wird auch die Unterstützung durch den Arbeitgeber für das freiwillige Engagement von Arbeitnehmerinnen und Arbeitnehmern betrachtet: Wer wird bei der Ausübung des freiwilligen Engagements durch den Arbeitgeber unterstützt und ist diese Unterstützung gewünscht? 


\subsection{Datengrundlage}

Die in diesem Kapitel berichteten deskriptiven Befunde basieren auf gewichteten Analysen. Für die Gewichtung wurden in allen Erhebungsjahren dieselben Gewichtungsmerkmale berücksichtigt. Dabei handelt es sich um die Designgewichtung, die sich auf die Auswahlwahrscheinlichkeit einer Person bezieht (Haushaltsgröße, Zahl der Festnetznummern und, nur für das Jahr 2014, auch die Zahl der Mobilfunknummern) und Gewichtungsmerkmale, die Abweichungen der Stichprobenverteilung von der Grundgesamtheit in bestimmten Dimensionen ausgleichen (Bundesland, Gemeindegrößenklassen, Geschlecht, Altersgruppen). Von einer Hinzunahme weiterer Gewichtungsmerkmale wie etwa des Bildungsstandes haben wir abgesehen, um die Vergleichbarkeit mit den bisher veröffentlichten Berichten zu erhalten.

Organisatorischer Rahmen: Im Freiwilligensurvey 2014 wird erfasst, in welchem organisatorischen Rahmen Engagierte ihre zeitintensivste freiwillige Tätigkeit (bei Personen mit mehreren freiwilligen Tätigkeiten) oder ihre einzige freiwillige Tätigkeit ausüben:

In welchem organisatorischen Rahmen üben Sie Ihre Tätigkeit aus? Ist das ein Verein; ein Verband; eine Gewerkschaft; eine Partei; eine Kirche oder eine religiöse Vereinigung; eine Nachbarschaftshilfe; eine Initiative oder Projektarbeit; eine selbst organisierte Gruppe; eine staatliche oder kommunale Einrichtung; eine private Einrichtung; eine Stiftung; allein, nicht in einer Gruppe, Organisation oder Einrichtung; sonstiges?

Die Frage zum organisatorischen Rahmen der freiwilligen Tätigkeit wurde in allen Wellen des Freiwilligensurveys gestellt. Es gab allerdings jeweils geringfügige Modifikationen der Antwort- möglichkeiten, die wir zu fünf Organisationsformen zusammengefasst haben: 1) Im Rahmen von Vereinen oder Verbänden, 2) in Kirchen oder religiösen Vereinigungen, 3) bei staatlichen oder kommunalen Einrichtungen, 4) in anderen überwiegend formal organisierten Einrichtungen oder 5) individuell organisiert, außerhalb des formalen Rahmens einer Institution in selbst organisierten Gruppen (Vergleiche die Übersicht in Tabelle 20-1). Wir betrachten Engagement in Vereinen und Verbänden gemeinsam, weil diese juristische Aufteilung aus Sicht der Engagierten oftmals nicht trennscharf ist. Weitere formale Organisationstypen sind Kirchen oder religiöse Vereinigungen, staatliche oder kommunale Einrichtungen sowie andere überwiegend formal organisierte Einrichtungen (die Kategorie ,Sonstiges' könnte auch nicht formal organisierte Einrichtungen enthalten, sie ist jedoch vernachlässigbar gering besetzt).

Individuell organisierte Gruppen umfassen das Engagement in Selbsthilfegruppen (2014 aufgrund eines Fehlers bei der Fragebogenkonstruktion nicht erhoben) beziehungsweise Nachbarschaftshilfen (2014 neu eingeführt) ebenso wie das Engagement in Initiativen oder Projekten, das Engagement in selbst organisierten Gruppen und das Engagement, dass alleine ausgeübt wird (seit 2009 erhoben). Es ist davon auszugehen, dass die Vergleichbarkeit der gruppierten Ergebnisse über die Erhebungswellen gewährleistet ist, da Engagierte in Selbsthilfegruppen bei der Befragung 2014 sehr wahrscheinlich die Antwortkategorien ,Selbst organisierte Gruppe` oder aber ,Initiative oder Projekt' wählten, und somit wie in den Jahren zuvor zur übergeordneten Organisationsform der individuell organisierten Gruppe zählen. 
Tabelle 20-1: Organisationsformen des freiwilligen Engagements

\begin{tabular}{|c|c|c|c|c|c|}
\hline & Organisationsform & 1999 & 2004 & 2009 & 2014 \\
\hline \multirow{9}{*}{ 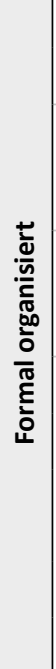 } & \multirow{2}{*}{$\begin{array}{l}\text { Vereine oder } \\
\text { Verbände }\end{array}$} & Verein & Verein & Verein & Verein \\
\hline & & Verband & Verband & Verband & Verband \\
\hline & $\begin{array}{l}\text { Kirche oder } \\
\text { religiöse } \\
\text { Vereinigungen }\end{array}$ & $\begin{array}{l}\text { Kirche oder } \\
\text { religiöse } \\
\text { Vereinigung }\end{array}$ & $\begin{array}{l}\text { Kirche oder } \\
\text { religiöse } \\
\text { Vereinigung }\end{array}$ & $\begin{array}{l}\text { Kirche oder } \\
\text { religiöse } \\
\text { Vereinigung }\end{array}$ & $\begin{array}{l}\text { Kirche oder } \\
\text { religiöse } \\
\text { Vereinigung }\end{array}$ \\
\hline & $\begin{array}{l}\text { Staatliche oder } \\
\text { kommunale } \\
\text { Einrichtungen }\end{array}$ & $\begin{array}{l}\text { Staatliche oder } \\
\text { kommunale } \\
\text { Einrichtung }\end{array}$ & $\begin{array}{l}\text { Staatliche oder } \\
\text { kommunale } \\
\text { Einrichtung }\end{array}$ & $\begin{array}{l}\text { Staatliche oder } \\
\text { kommunale } \\
\text { Einrichtung }\end{array}$ & $\begin{array}{l}\text { Staatliche oder } \\
\text { kommunale } \\
\text { Einrichtung }\end{array}$ \\
\hline & \multirow{5}{*}{$\begin{array}{l}\text { Andere formal } \\
\text { organisierte } \\
\text { Einrichtungen }\end{array}$} & Gewerkschaft & Gewerkschaft & Gewerkschaft & Gewerkschaft \\
\hline & & Partei & Partei & Partei & Partei \\
\hline & & \multirow{2}{*}{$\begin{array}{l}\text { Private Ein- } \\
\text { richtung oder } \\
\text { Stiftung }\end{array}$} & $\begin{array}{l}\text { Private } \\
\text { Einrichtung }\end{array}$ & $\begin{array}{l}\text { Private } \\
\text { Einrichtung }\end{array}$ & $\begin{array}{l}\text { Private } \\
\text { Einrichtung }\end{array}$ \\
\hline & & & Stiftung & Stiftung & Stiftung \\
\hline & & Sonstiges & Sonstiges & Sonstiges & Sonstiges \\
\hline \multirow{5}{*}{ 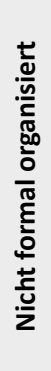 } & \multirow{5}{*}{$\begin{array}{l}\text { Individuell } \\
\text { organisierte } \\
\text { Gruppen }\end{array}$} & $\begin{array}{l}\text { Selbsthilfe- } \\
\text { gruppe }\end{array}$ & $\begin{array}{l}\text { Selbsthilfe- } \\
\text { gruppe }\end{array}$ & $\begin{array}{l}\text { Selbsthilfe- } \\
\text { gruppe }\end{array}$ & (nicht erhoben) \\
\hline & & (nicht erhoben) & (nicht erhoben) & (nicht erhoben) & Nachbarschaftshilfe \\
\hline & & $\begin{array}{l}\text { Initiative oder } \\
\text { Projekt }\end{array}$ & $\begin{array}{l}\text { Initiative oder } \\
\text { Projekt }\end{array}$ & $\begin{array}{l}\text { Initiative oder } \\
\text { Projekt }\end{array}$ & $\begin{array}{l}\text { Initiative oder } \\
\text { Projekt }\end{array}$ \\
\hline & & $\begin{array}{l}\text { Selbst organisierte } \\
\text { Gruppe }\end{array}$ & $\begin{array}{l}\text { Selbst organisierte } \\
\text { Gruppe }\end{array}$ & $\begin{array}{l}\text { Selbst organisierte } \\
\text { Gruppe }\end{array}$ & $\begin{array}{l}\text { Selbst organisierte } \\
\text { Gruppe }\end{array}$ \\
\hline & & (nicht erhoben) & (nicht erhoben) & Allein & Allein \\
\hline
\end{tabular}

Quelle: FWS 1999, FWS 2004, FWS 2009, FWS 2014: „In welchem organisatorischen Rahmen üben Sie Ihre Tätigkeit aus? Ist das..." Nachrichtlich: Die Kategorie Selbsthilfegruppe wurde 2014 aufgrund eines Fehlers bei der Fragebogenkonstruktion nicht erhoben. Eigene Darstellung (DZA).

Ansprechpartner und Mitsprachemöglichkeiten: Über die Art der Organisation hinaus, in denen Engagierte ihr Engagement ausüben, ist im Freiwilligensurvey 2014 erfragt, ob es dort eine Ansprechpartnerin oder einen Ansprechpartner für sie gibt und wie die Mitsprachemöglichkeiten in der jeweiligen Organisation bewertet werden:

Gibt es in [diesem/dieser] [Verein/Verband; Gewerkschaft; Partei; Kirche oder religiöse Vereinigung; Nachbarschaftshilfe; Projektarbeit; selbst organisierte Gruppe; staatliche oder kommunale Einrichtung; private Einrichtung; Stiftung] einen
Ansprechpartner oder eine Ansprechpartnerin, der oder die sich speziell um die Ehrenamtlichen oder Freiwilligen kümmert?

\section{Antwortmöglichkeiten: ja; nein}

Wie bewerten Sie in [diesem/dieser] [Verein/Verband; Gewerkschaft; Partei; Kirche oder religiöse Vereinigung; Nachbarschaftshilfe; Projektarbeit; selbst organisierte Gruppe; staatliche oder kommunale Einrichtung; private Einrichtung; Stiftung] Ihre Möglichkeiten zur Mitsprache und Mitentscheidung? 
Antwortmöglichkeiten: sehr gut; eher gut; teils/ teils; eher schlecht; sehr schlecht

Beim Vorlesen der Fragen wird im computerunterstützten Telefoninterview die jeweilige Organisation eingeblendet, die die befragte Person vorher bei der Frage zum organisatorischen Rahmen genannt hat.

Verbesserungsmöglichkeiten: Engagierte können ihre Arbeiten und Aufgaben besser ausüben, wenn die Rahmenbedingungen dafür stimmen und sie von Seiten der Organisationen und des Staates, aber auch von anderen wie beispielsweise ihren Arbeitgebern in ihrem Engagement unterstützt werden.

Im Freiwilligensurvey 2014 werden folgende Angaben $\mathrm{zu}$ den Verbesserungsmöglichkeiten seitens der Organisationen erfragt:

Es wird viel darüber diskutiert, mit welchen Maßnahmen man ehrenamtliches oder freiwilliges Engagement fördern und unterstützen könnte. Zunächst zu der Frage, was die Organisationen selbst tun könnten. Wenn Sie an Ihre eigene Tätigkeit denken, bei welchen der folgenden Punkte würden Sie sagen: da wären Verbesserungen wichtig? Bei der fachlichen Unterstützung der Tätigkeit; bei den Weiterbildungsmöglichkeiten; bei der Anerkennung der Tätigkeit durch hauptamtliche Kräfte in der Organisation; bei der finanziellen Vergütung für die geleistete Arbeit; bei einer unbürokratischen Kostenerstattung; bei der Anerkennung der Tätigkeit in Form von Zeugnissen, Ausweisen oder Ähnlichem; bei der Bereitstellung von geeigneten Räumen und Ausstattungsmitteln für die Projektund Gruppenarbeit?

Antwortkategorien sind jeweils: ja, trifft zu; nein, trifft nicht $z u$

Darüber hinaus wird erfasst, wo die Engagierten Verbesserungsmöglichkeiten durch den Staat und die Öffentlichkeit sehen:

Andere Vorschläge zur Förderung ehrenamtlichen oder freiwilligen Engagements richten sich an den
Staat und die Öffentlichkeit. Denken Sie bitte wieder an Ihre eigene Tätigkeit und Ihre persönliche Situation. Bei welchen der folgenden Punkte würden Sie sagen: Da wären Verbesserungen wichtig? Bei der Absicherung durch Haftpflicht- und Unfallversicherung; bei der steuerlichen Absetzbarkeit von Kosten; bei der steuerlichen Freistellung von Aufwandsentschädigungen; bei der Anerkennung ehrenamtlicher Tätigkeit als berufliches Praktikum oder als berufliche Weiterbildung; bei der öffentlichen Anerkennung in Form von Ehrungen und Ähnlichem; bei der besseren Information und Beratung über Gelegenheiten zum ehrenamtlichen oder freiwilligen Engagement; bei der Vereinbarkeit mit dem Beruf?

Antwortkategorien sind jeweils: ja,trifft zu; nein, trifft nicht $z u$

Unterstützung durch den Arbeitgeber: Für abhängig beschäftigte Engagierte können in Bezug auf die Ausübung ihrer freiwilligen Tätigkeit deren Arbeitgeber mehr oder minder unterstützend sein. Erfasst wird dies im Freiwilligensurvey 2014 durch die folgende Abfrage:

Und nun noch eine Frage zu Ihrem Arbeitgeber. Unterstützt Sie dieser bei Ihrem freiwilligen Engagement, zum Beispiel durch flexible Arbeitszeiten oder Bereitstellen von Räumen?

Antwortmöglichkeiten: ja; nein

Bei Verneinung schließt sich diese Folgefrage an:

Möchten Sie, dass Ihr Arbeitgeber Sie bei Ihrem freiwilligen Engagement unterstützt?

Antwortmöglichkeiten: ja; nein 


\subsection{Organisationsformen des Engagements}

\subsubsection{Organisationsformen 2014}

Freiwilliges Engagement findet anteilig am häufigsten in Vereinen und Verbänden statt. Über die Hälfte (52,1 Prozent) der Engagierten übt ihre freiwillige Tätigkeit in einem Verein oder einem Verband aus (Abbildung 20-1a). Dies entspricht etwa 15,8 Millionen Personen. ${ }^{1}$ Anteilig deutlich seltener ist freiwilliges Engagement in einer Kirche oder religiösen Vereinigung (13,0 Prozent der Engagierten beziehungsweise 3,9 Millionen Menschen). In einer staatlichen oder kommunalen Einrichtung sind 8,7 Prozent der Engagierten freiwillig tätig. Dies entspricht ungefähr 2,6 Millionen Menschen. Ein Zehntel der Engagierten übernimmt Aufgaben in einer anderen überwiegend formal organisierten Einrichtung wie einer Partei oder einer Stiftung. Dies entspricht etwa 3,1 Millionen Personen. In individuell organisierten Gruppen engagieren sich 16,0 Prozent aller Engagierten (4,9 Millionen Menschen).

Geschlechterunterschiede zeigen sich insbesondere hinsichtlich des Engagements in Vereinen und Verbänden - deutlich mehr Männer als Frauen sind in dieser Organisationsform engagiert. Zudem sind Männer häufiger als Frauen Mitglied in einem Verein (siehe Kapitel 8). Auch in Bezug auf das Engagement in Kirchen und religiösen Vereinigungen sind die Unterschiede deutlich. Hier engagieren sich anteilig wesentlich mehr Frauen als Männer. Frauen sind anteilig auch etwas häufiger in individuell organisierten Gruppen engagiert. Personen ab 65 Jahren engagieren sich anteilig seltener als andere Altersgrup-

1 Zahlen auf Individual- und Organisationsebene korrespondieren: Dem Survey Zivilgesellschaft in Zahlen (ZiviZ) zufolge zählen gemeinnützige Organisationen 17,5 Millionen ,Engagements, wobei der ganz überwiegende Teil auf Vereine entfällt. Da es vorkommen kann, dass sich eine Person in mehreren Organisationen engagiert, bezieht sich die Angabe dabei auf freiwillige Tätigkeiten, nicht auf Engagierte (Krimmer \& Priemer 2013: 37). pen in Vereinen und zu etwas höheren Anteilen in kirchlichen oder religiösen Organisationen.

Hinsichtlich der Organisationsformen des Engagements unterscheiden sich die Bildungsgruppen nur geringfügig (Abbildung 20-1b). Schülerinnen und Schüler engagieren sich tendenziell zu geringeren Anteilen in Vereinen oder Verbänden und zu höheren Anteilen in Kirchen oder religiösen Vereinigungen (18,4 Prozent, bei den anderen Bildungsgruppen liegen die Anteile jeweils bei maximal 13 Prozent). Höhergebildete engagieren sich dafür zu etwas höheren Anteilen in individuell organisierten Gruppen (17,3 Prozent). Bei den Schülerinnen und Schülern beträgt dieser Anteil 15,1 Prozent, bei den Niedriggebildeten 14,2 Prozent und bei den Personen mit mittlerer Schulbildung 14,9 Prozent.

Somit unterscheiden sich die Organisationsformen bezüglich der Bevölkerungsgruppen, die in dem ein oder anderen organisatorischen Rahmen anteilig häufiger tätig werden. Darüber hinaus unterscheiden sie sich auch in Hinblick auf die gesellschaftlichen Bereiche, in denen die freiwillig Engagierten ihre Tätigkeiten ausüben. Beinahe die Hälfte des Engagements in Vereinen und Verbänden entfällt auf den Bereich Sport und Bewegung (Tabelle 20-2 im Online-Anhang). Vereine und Verbände prägen den Sport demnach besonders stark. Knapp zwei Drittel des Engagements in Kirchen oder religiösen Vereinigungen entfällt auf den kirchlichen oder religiösen Bereich. Beim Engagement im Rahmen von staatlichen oder kommunalen Einrichtungen sind die größten gesellschaftlichen Bereiche neben Schule und Kindergarten der Unfall- oder Rettungsdienst und die Freiwillige Feuerwehr und der soziale Bereich. Der kleine gesellschaftliche Bereich Justiz und Kriminalitätsprobleme, der überwiegend von staatlicher Seite organisiert ist, macht 3,5 Prozent des Engagements in staatlichen oder kommunalen Einrichtungen aus. 
Abbildung 20-1: Organisationsformen des freiwilligen Engagements 2014, a) gesamt, nach Geschlecht und nach Alter sowie b) nach Bildung

a) gesamt, nach Geschlecht und nach Alter

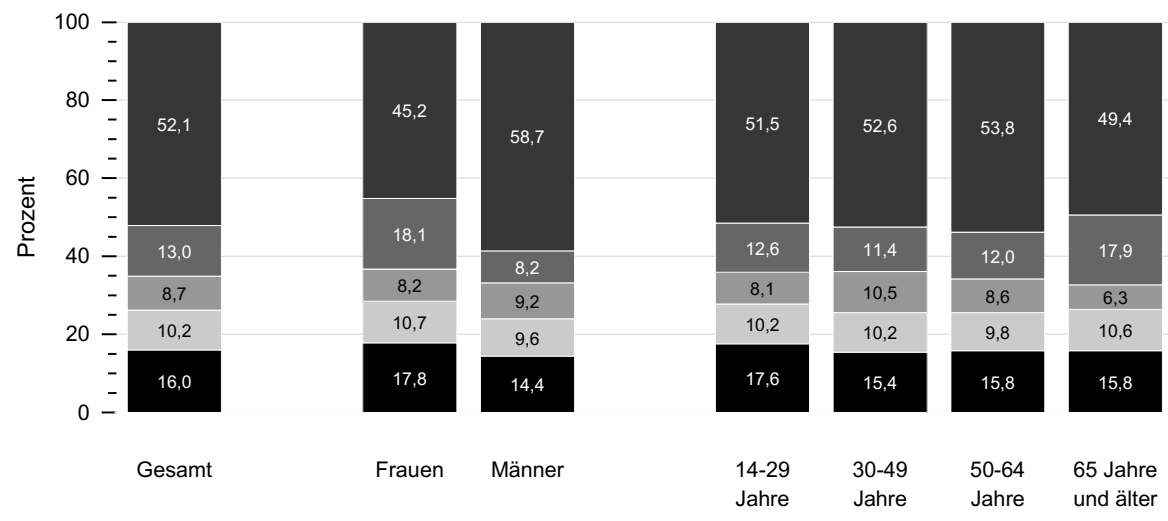

b) nach Bildung

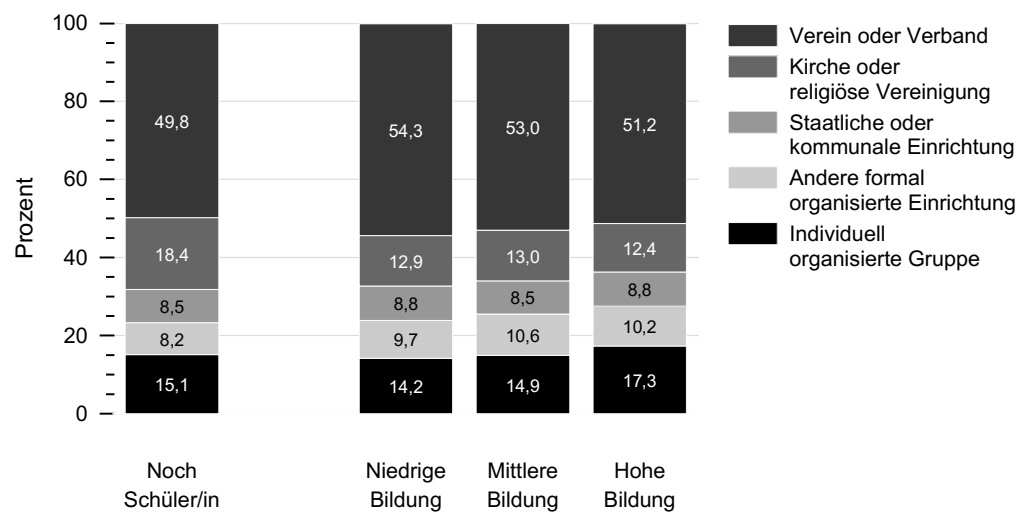

Quelle: FWS 2014, gewichtet, eigene Berechnungen (DZA). Basis: Alle Engagierten. Gesamt, Geschlecht und Alter $(n=12.296)$, Bildung $(n=12.293)$.

Das individuell organisierte Engagement unterscheidet sich von diesen stärker formalen Organisationsformen bezüglich der gesellschaftlichen Tätigkeitsfelder insbesondere durch seine große Vielfalt: Obwohl auch die in individuell organisierten Gruppen Engagierten in allen vierzehn gesellschaftlichen Bereichen tätig sind, überwiegen die Bereiche Soziales, Kultur und Musik, Schule und Kindergarten sowie Freizeit und Geselligkeit. Insgesamt ist jedoch festzustellen, dass alle Organisationsformen für jeweils recht vielfältiges Engagement in unterschiedlichen Bereichen stehen.

\subsubsection{Organisationsformen im Zeitvergleich}

Freiwilliges Engagement findet heute $\mathrm{zu}$ einem vergleichsweise geringeren Anteil im Rahmen von Vereinen oder Verbänden statt als in der 
Vergangenheit (Abbildung 20-2): 1999 übten 55,8 Prozent aller Engagierten ihre freiwillige Tätigkeit in einem Verein oder einem Verband aus. 2004 taten dies 56,3 Prozent. 2009 waren es 53,4 Prozent und 2014 üben 52,1 Prozent der Engagierten ihre Tätigkeit in einem Verein oder Verband aus. Das Engagement im Rahmen von Kirchen oder religiösen Vereinigungen bleibt stabil: Im Jahr 1999 engagierten sich dort 13,6 Prozent, 2004 lag der Anteil bei 14,4 Prozent, 2009 bei 14,3 Prozent und 2014 liegt er bei 13,0 Prozent.

Der Anteil an Engagierten, die ihr Engagement außerhalb formal organisierter zivilgesellschaftlicher Einrichtungen ausüben, nämlich in
Form des individuell organisierten Engagements, etwa in Initiativen, Projekten und selbstorganisierten Gruppen, hat zugenommen. So stieg der Anteil an allen Engagierten von 11,0 Prozent im Jahr 1999 und 10,1 Prozent im Jahr 2004 auf 14,6 Prozent im Jahr 2009 und 16,0 Prozent im Jahr 2014. Zudem findet freiwilliges Engagement vergleichsweise seltener im Rahmen staatlicher oder kommunaler Einrichtungen statt als früher. Dieser Anteil freiwillig Engagierter ist von 10,2 Prozent im Jahr 1999 und im Jahr 2004 auf 9,2 Prozent im Jahr 2009 und 8,7 Prozent im Jahr 2014 gesunken.

\section{Abbildung 20-2: Organisationsformen des freiwilligen Engagements im Zeitvergleich}

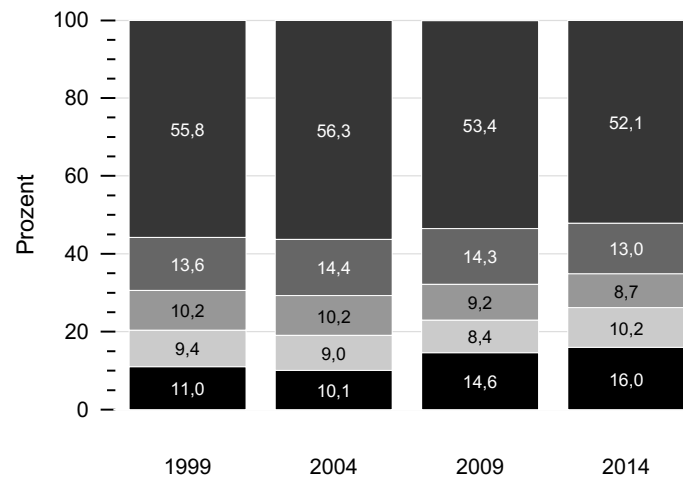

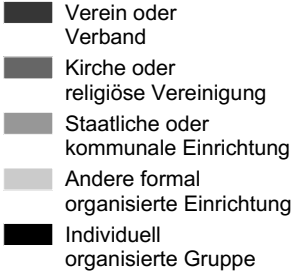

organisierte Gruppe

Quelle: FWS, gewichtet, eigene Berechnungen (DZA). Basis: Alle Engagierten. FWS 1999 ( $n=4.683$ ), FWS 2004

$(n=5.128)$, FWS $2009(n=6.986), 2014(n=12.296)$

Die hier beschriebenen Befunde - sinkender Anteil des freiwilligen Engagements in Vereinen und Verbänden versus steigender Anteil des individuell organisierten Engagements - basieren auf der Betrachtung aller Engagierten (Abbildung 20-2). Setzen wir diese Befunde jedoch ins Verhältnis zur gesamten Wohnbevölkerung im Alter ab 14 Jahren, ist festzustellen, dass der relative Rückgang des Vereins- und Verbandsengagements nicht mit einem Rückgang der absoluten Zahl von Engagierten einhergeht. Der relative Rückgang ist allein der Tatsache geschuldet, dass individuell organisiertes freiwilliges Engagement in deutlich stärkerem Maße gestiegen ist als das Engagement in Vereinen und Verbänden.

Insgesamt ist der Anteil aller freiwillig Engagierten an der Wohnbevölkerung von 34,0 Prozent im Jahr 1999 auf 43,6 Prozent im Jahr 2014 gestiegen (siehe Kapitel 3). Von den 34,0 Prozent waren mit 55,8 Prozent mehr als die Hälfte der Engagierten bei Vereinen oder Verbänden tätig, das entspricht einem Anteil an der Gesamtbevölkerung von 19,0 Prozent. Im Jahr 2014 liegt der Anteil an der Gesamtbevölkerung bereits bei 22,7 Prozent (beziehungsweise 52,1 Prozent der insgesamt 43,6 Prozent Engagierten). 
Was bei der Betrachtung der Verteilung aller freiwillig Engagierten auf die verschiedenen Organisationsformen nicht sichtbar wird: Tatsächlich hat das Engagement in allen Organisationsformen im Zeitvergleich zugenommen, jedoch in jeweils unterschiedlichem Ausmaß. Am deutlichsten ist das individuell organisierte Engagement gewachsen, denn das Engagement in individuell organisierten Gruppen hat in der Wohnbevölkerung ab 14 Jahren von 3,8 Prozent im Jahr 1999 auf 7,0 Prozent im Jahr 2014 zugenommen (ohne Abbildung). Gleichzeitig ist aber auch das Engagement in Vereinen und Verbän- den deutlich gestiegen von 19,0 Prozent im Jahr 1999 auf 22,7 Prozent im Jahr 2014. Ebenso hat das Engagement in anderen überwiegend formal organisierten Einrichtungen etwas zugenommen von 3,2 Prozent im Jahr 1999 auf 4,4 Prozent im Jahr 2014. Lediglich leicht angestiegen ist das Engagement in Kirchen oder religiösen Vereinigungen, von 4,6 Prozent im Jahr 1999 auf 5,7 Prozent im Jahr 2014. Das Engagement in staatlichen oder kommunalen Einrichtungen hat kaum zugenommen von 3,5 Prozent im Jahr 1999 auf 3,8 Prozent im Jahr 2014.

\subsection{Organisationale Rahmenbedingungen des Engagements}

Die Organisationsformen sind für die Ausgestaltung des freiwilligen Engagements auch deswegen bedeutsam, weil sie für unterschiedliche zivilgesellschaftliche Akteure stehen, die unterschiedliche Rahmenbedingungen für freiwilliges Engagement schaffen können. So sind beispielsweise größere Organisationen eher in der Lage als kleinere, eine Ansprechpartnerin oder einen Ansprechpartner bereitzustellen, die oder der sich um die Belange der Engagierten kümmert. Möglicherweise besteht in diesen Organisationen auch mehr Bedarf für eine Ansprechperson, um die Kommunikation zwischen freiwilligen und hauptamtlichen Kräften zu sichern. Darüber hinaus wirken sich unterschiedliche Strukturierungs- und Hierarchiegrade auf die Möglichkeiten der Mitsprache aus. Im Folgenden wird betrachtet, ob es in den Organisationen eine Ansprechpartnerin beziehungsweise einen Ansprechpartner für die Engagierten gibt und wie die Möglichkeiten zur Mitsprache bewertet werden. Im Zentrum steht dabei die Frage, wie sich diese Rahmenbedingungen zwischen den Organisationsformen unterscheiden. 


\subsubsection{Vorhandensein einer Ansprechpartnerin oder eines Ansprechpartners}

Von den Engagierten ist die Mehrheit (63,2 Prozent) in Organisationen freiwillig tätig, in denen eine Ansprechpartnerin oder ein Ansprechpartner für sie vorhanden ist (Abbildung 20-3). Dabei unterscheiden sich die Organisationsformen jedoch recht deutlich voneinander. Rund drei Viertel der Engagierten, die sich in religiösen Vereinigungen engagieren, berichten, dass es dort eine Ansprechpartnerin oder einen Ansprechpartner für sie gibt, gleiches gilt bei staatlichen oder kommunalen Einrichtungen. Von den Engagierten, die sich in individuell organisierten Gruppen engagieren, berichtet dies knapp die Hälfte.

\section{Abbildung 20-3: Vorhandensein einer Ansprechpartnerin oder eines Ansprechpartners 2014, nach Organisationsform}

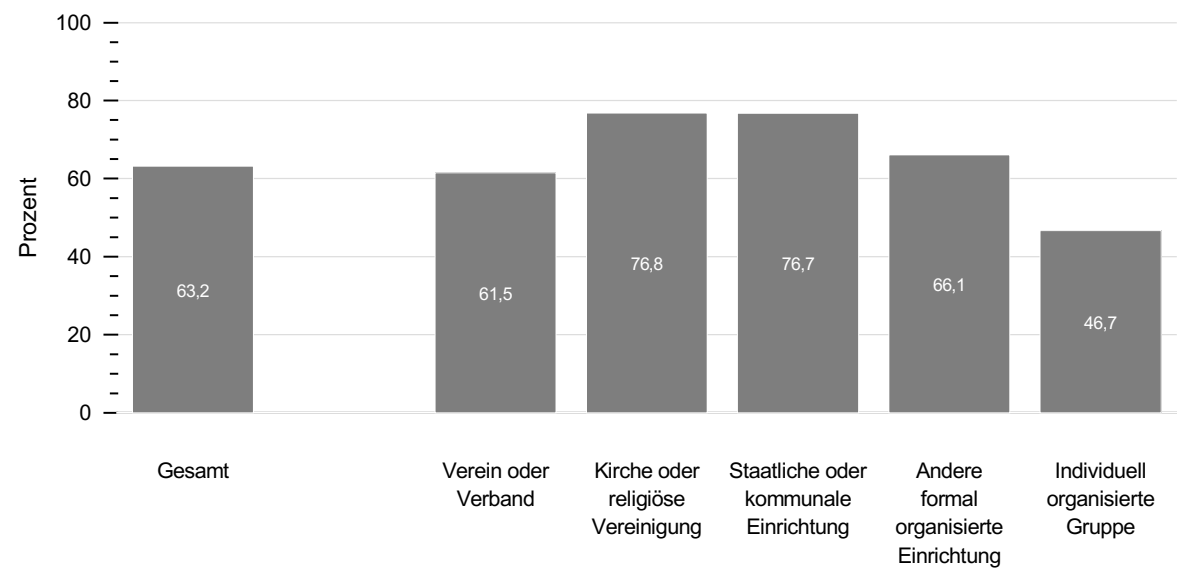

Quelle: FWS 2014, gewichtet, eigene Berechnungen (DZA). Basis: Alle Engagierten, die sich im Rahmen einer dieser Organisationen engagieren: Verein/Verband/Gewerkschaft/Partei/Kirche oder religiöse Vereinigung/Nachbarschaftshilfe/Projektarbeit/Selbst organisierte Gruppe/Staatliche oder kommunale Einrichtung/Private Einrichtung/Stiftung $(n=11.170)$.

Frauen berichten anteilig etwas häufiger als Männer, dass es in der Organisation, in der sie sich engagieren, eine Ansprechpartnerin oder einen Ansprechpartner gibt (64,8 Prozent zu 61,6 Prozent). Dieser Unterschied ist bei den individuell organisierten Gruppen besonders ausgeprägt: Hier berichten 51,2 Prozent der Frauen von einer Ansprechperson, aber nur 41,8 Prozent der Männer; Frauen und Männer engagieren sich offenbar in unterschiedlich strukturierten Gruppen. Eine umgekehrte Tendenz ist bei staatlichen und kommunalen Einrichtungen zu beobachten, bei denen mehr Männer als Frauen berichten, dass eine Ansprechperson für die freiwillig Engagierten zur Verfügung steht (Tabelle 20-3 im Online-Anhang).

Bei der Betrachtung nach Alter ist zu erkennen, dass vor allem jüngere (14 bis 29 Jahre) und ältere (65 und mehr Jahre) Engagierte sich in Organisationen engagieren, in denen eine Ansprechpartnerin oder ein Ansprechpartner für sie zur Verfügung steht. Bei Personen mittleren Alters ist dies anteilig seltener der Fall. Vor allem in Kirchen und religiösen Gruppen (83,3 Prozent) sowie in staatlichen und kommunalen Einrichtungen (79,7 Prozent) finden 14- bis 29-jährige 
Engagierte besonders häufig eine Ansprechperson vor (Tabelle 20-3 im Online-Anhang).

Im Zeitverlauf betrachtet (Abbildung 20-4) hat sich hinsichtlich des Vorhandenseins einer Ansprechperson nicht viel verändert. Im Jahr 2004 war in 65,4 Prozent der Organisationen eine entsprechende Person vorhanden, 2009 in 62,5 Prozent und 2014 sind es 63,2 Prozent. Dabei gab es eine leichte Abnahme bei den Vereinen und Verbänden (von 64,7 Prozent im Jahr 2004 auf 61,5 Prozent im Jahr 2014) und einen relativ deutlichen Rückgang (von 52,1 Prozent im Jahr 2004 auf 46,7 Prozent im Jahr 2014) bei den individuell organisierten Gruppen. Bei den Kirchen und religiösen Vereinigungen ebenso wie bei den staatlichen und kommunalen Einrichtungen bleiben die Anteile auf hohem Niveau relativ stabil. Andere Organisationsformen wie Gewerkschaften, Parteien oder Stiftungen verzeichnen einen deutlichen Zuwachs (von 57,2 Prozent 2004 auf 66,1 Prozent im Jahr 2014).

\section{Abbildung 20-4: Vorhandensein einer Ansprechpartnerin oder eines Ansprechpartners im Zeitvergleich, nach Organisationsform}

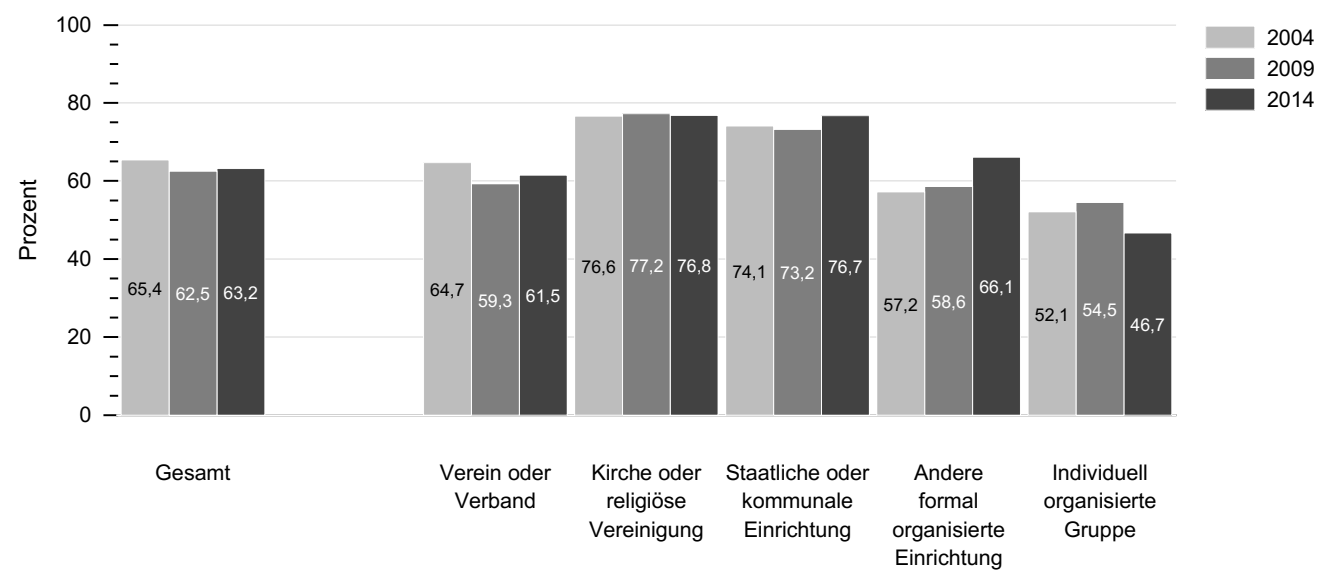

Quelle: FWS 2004 ( $n=5.016)$, FWS 2009 ( $n=6.662$ ), FWS $2014(n=11.170)$, gewichtet, eigene Berechnungen (DZA). Basis: Alle Engagierten, die sich im Rahmen einer dieser Organisationen engagieren: Verein/Verband/Gewerkschaft/ Partei/Kirche oder religiöse Vereinigung/Nachbarschaftshilfe/Projektarbeit/Selbst organisierte Gruppe/Staatliche oder kommunale Einrichtung/Private Einrichtung/Stiftung. Bewertung der Mitsprachemöglichkeiten. Diese Information wurde 1999 nicht erhoben. 


\subsubsection{Bewertung der Mitsprache- möglichkeiten}

Die Mitsprachemöglichkeiten in den Organisationen werden überwiegend als gut oder sehr gut bewertet. Nur eine sehr kleine Minderheit hält die Mitsprachemöglichkeiten für schlecht oder sehr schlecht (Abbildung 20-5). Dabei werden die Mitsprachemöglichkeiten in den individuell organisierten Gruppen anteilig am häufigsten als gut oder sehr gut bewertet, in den staatlichen oder kommunalen Einrichtungen hingegen am seltensten.

Männer bewerten die Mitsprachemöglichkeiten insgesamt etwas besser als Frauen (Tabelle 20-4 im Online-Anhang). Dies könnte auch da- mit zusammenhängen, dass deutlich mehr Männer eine Leitungsfunktion im Engagement haben als Frauen und daher über bessere Mitsprachemöglichkeiten verfügen. Bei den Vereinen und Verbänden, in den kirchlichen und religiösen Vereinigungen sowie in den staatlichen und kommunalen Einrichtungen bewerten anteilig mehr Männer die Mitsprachemöglichkeiten als eher gut oder sehr gut, dagegen bewerten sie bei den anderen Organisationsformen die Mitsprachemöglichkeiten etwas seltener als eher schlecht oder sehr schlecht. Lediglich bei individuell organisierten Gruppen fällt die Bewertung der Mitsprachemöglichkeiten durch Männer und Frauen nahezu gleich aus.

\section{Abbildung 20-5: Bewertung der Mitsprachemöglichkeiten 2014, nach Organisationsform}

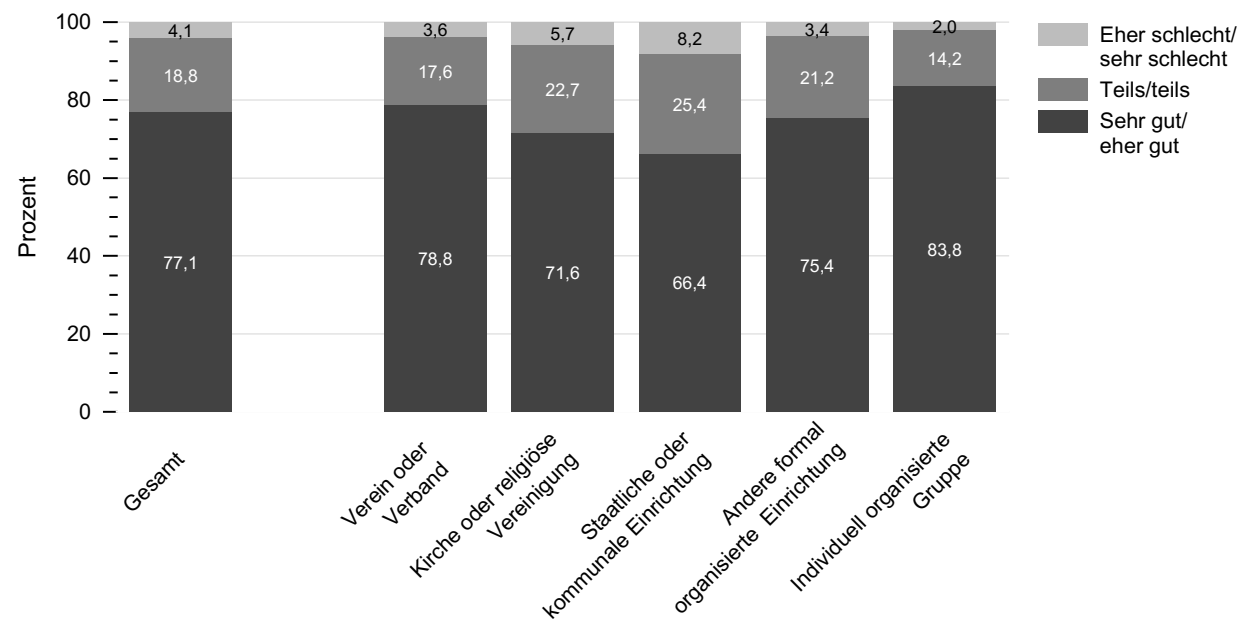

Quelle: FWS 2014, gewichtet, eigene Berechnungen (DZA). Basis: Alle Engagierten, die sich im Rahmen einer dieser Organisationen engagieren: Verein/Verband/Gewerkschaft/Partei/Kirche oder religiöse Vereinigung/Nachbarschaftshilfe/Projektarbeit/Selbst organisierte Gruppe/Staatliche oder kommunale Einrichtung/Private Einrichtung/Stiftung $(n=11.344)$.

Nach Altersgruppen betrachtet zeigt sich ein heterogenes Bild (Tabelle 20-4 im Online-Anhang): In den Vereinen und Verbänden sowie in den staatlichen und kommunalen Einrichtungen bewerten Ältere im Vergleich zu Jüngeren die Mitsprachemöglichkeiten zu größeren Anteilen als gut oder sehr gut. Auch bei den sonstigen Organisationsformen findet sich dieses Muster, allerdings mit Ausnahme der jüngsten Altersgruppe, die die Mitsprachemöglichkeiten hier vergleichbar positiv wie die älteste Altersgruppe bewertet. In den individuell organisierten Grup- 
pen sind die Ältesten etwas unzufriedener mit den Mitsprachemöglichkeiten als die jüngeren Altersgruppen und bei den Kirchen und religiösen Einrichtungen bewertet die jüngste Altersgruppe die Mitsprachemöglichkeiten etwas kritischer als die übrigen Altersgruppen. Insgesamt überwiegen auch nach Altersgruppen betrachtet die Gemeinsamkeiten, die sich in der größtenteils positiven Bewertung von Mitsprachemöglichkeiten zeigen.

Von Engagierten mit unterschiedlicher Bildung werden ihre Mitsprachemöglichkeiten auch unterschiedlich bewertet: In Vereinen und Verbänden bewerten Engagierte mit hoher Bildung diese häufiger als sehr gut oder eher gut als Engagierte mit niedriger Bildung, und auch häufiger als Schülerinnen und Schüler (Tabelle 20-5 im Online-Anhang). Denselben Bildungsunterschied finden wir bei den individuell organisierten Gruppen, wenn auch auf einem deutlich höheren Niveau, da hier Mitsprachemöglichkeiten insgesamt am besten bewertet werden. In den anderen Organisationsformen bewerten Personen mit hoher Bildung ihre Mitsprachemöglichkeiten jedoch nicht häufiger mit gut als Personen mit mittlerer oder niedriger Bildung, etwa in Kirche oder religiösen Vereinigungen. In den staatlichen oder kommunalen Einrichtungen sind es Engagierte mit niedriger Bildung, die ihre Mitsprachemöglichkeiten am besten bewerten.

Engagierte, die als freiwillige Tätigkeit eine Leitungs- oder Vorstandsfunktion ausüben, bewerten ihre Mitsprachemöglichkeiten in der Tat häufiger als sehr gut oder eher gut als Engagierte ohne Leitungs- oder Vorstandsfunktion. Am häufigsten als eher schlecht oder schlecht bewerten Engagierte ohne eine solche Leitungs- oder Vorstandsfunktion ihre Mitsprachemöglichkeiten in staatlichen oder kommunalen Einrichtungen. Am geringsten ist der Unterschied zwischen Engagierten mit Leitungs- oder Vorstandsfunktion und ohne eine solche Funktion in den individuell organisierten Gruppen. Da diese weniger formal organisiert sind, spielen solche offiziellen Funktionen hier offensichtlich eine geringere Rolle.

Wie bedeutsam das Vorhandensein einer Ansprechpartnerin oder eines Ansprechpartners für die freiwillig Engagierten ist, zeigt sich auch anhand diesen Befunds: Engagierte bewerten ihre Mitsprachemöglichkeiten vergleichsweise häufiger als sehr gut oder eher gut, wenn sie in ihrer Organisation eine Ansprechpartnerin oder einen Ansprechpartner haben. Dies wird besonders deutlich, wenn sie sich in der Kirche oder einer religiösen Vereinigung beziehungsweise in einer staatlichen oder kommunalen Einrichtung engagieren (Abbildung 20-6). Ansprechpartnerinnen und Ansprechpartner sind in diesen formalen Organisationen wichtig für die Mitsprachemöglichkeiten, wahrscheinlich, weil sie eine direkte Vermittlerrolle zwischen den Interessen der Organisation und den Interessen der einzelnen Engagierten einnehmen. Bei den individuell organisierten Gruppen aber auch bei den Vereinen und Verbänden ist die Rolle der Ansprechpartnerinnen und Ansprechpartner zumindest im Hinblick auf die Bewertung der Mitsprachemöglichkeiten durch die Engagierten jedoch unerheblich. 
Abbildung 20-6: Bewertung der Mitsprachemöglichkeiten 2014, nach Organisationsform und Vorhandensein einer Ansprechpartnerin oder eines Ansprechpartners
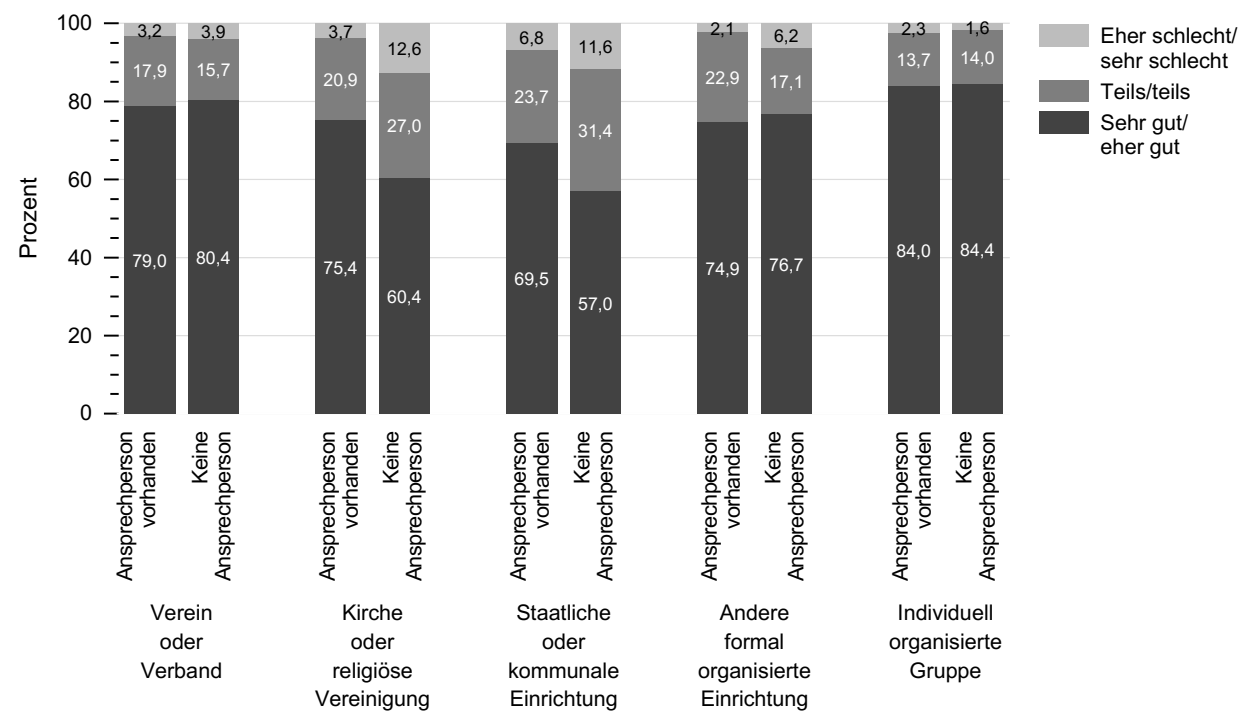

Quelle: FWS 2014, gewichtet, eigene Berechnungen (DZA). Basis: Alle Engagierten, die sich im Rahmen einer dieser Organisationen engagieren: Verein/Verband/Gewerkschaft/Partei/Kirche oder religiöse Vereinigung/Nachbarschaftshilfe/Projektarbeit/Selbst organisierte Gruppe/Staatliche oder kommunale Einrichtung/Private Einrichtung/Stiftung $(n=11.344)$.

\subsection{Verbesserungsmöglichkeiten im freiwilligen Engagement}

Engagierte können ihre Arbeiten und Aufgaben besser ausüben, wenn die Rahmenbedingungen dafür stimmen und sie von Seiten der Organisationen und des Staates, aber auch von anderen wie beispielsweise ihren Arbeitgebern in ihrem Engagement unterstützt werden.

\subsubsection{Verbesserungsmöglichkeiten durch die Organisationen}

Von ihren Organisationen wünschen sich die Engagierten insbesondere eine direkte Unterstützung bei ihren Aufgaben durch die Bereitstellung einer geeigneten Infrastruktur. Dies wird daran deutlich, dass die Engagierten mit Abstand am häufigsten Unterstützungsbedarf bei der Bereitstellung von geeigneten Räumen und Ausstattungsmitteln für die Projekt- und Gruppenarbeit nennen: 48,3 Prozent der Engagierten sehen hier Verbesserungsbedarf (Abbildung 20-7). Hierunter fallen auch finanzielle Mittel, die für die konkrete Durchführung der Projekte bereitgestellt werden sollen. Verbesserungsbedarf bei der fachlichen Unterstützung in ihrer freiwilligen Tätigkeit sehen 42,0 Prozent der Engagierten und bei den Weiterbildungsmöglichkeiten 40,8 Prozent. Lediglich jede beziehungsweise jeder fünfte Engagierte sieht Verbesserungsbedarf bei der finanziellen Vergütung für die geleistete Arbeit, die den Engagierten selbst zugutekommen würde. Das ist mit $\mathrm{Ab}$ stand der am seltensten genannte Bedarf. Das 
primäre Interesse der Engagierten scheint es, eine Verbesserung der Rahmenbedingungen ihrer Tätigkeit einzufordern, um ihre Sache oder Idee voranzubringen, und keinesfalls, ihre persönlichen Interessen etwa zur finanziellen Besserstellung einzufordern.

\section{Abbildung 20-7: Verbesserungsmöglichkeiten durch die Organisationen 2014}

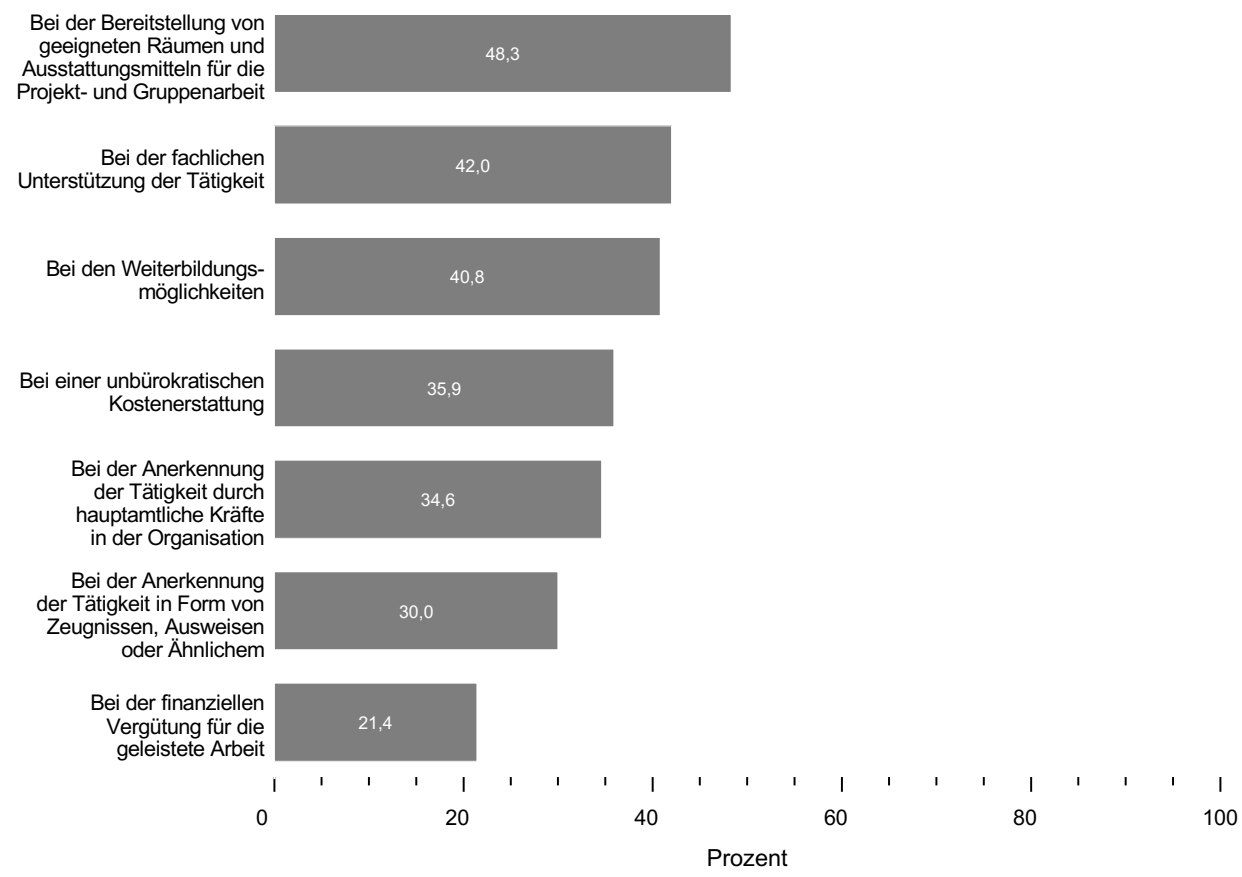

Quelle: FWS 2014, gewichtet, eigene Berechnungen (DZA). Basis: Alle Engagierten. Mehrfachnennungen möglich. Bereitstellung von Räumen ( $n=12.343)$, Fachliche Unterstützung $(n=12.339)$, Weiterbildung $(n=12.318)$, Kostenerstattung ( $n=12.240)$, Anerkennung durch Hauptamtliche $(n=12.253)$, Anerkennung durch Zeugnisse ( $n=12.327)$, Vergütung $(n=12.315)$.

Frauen und Männer, die sich freiwillig engagieren, schätzen die Verbesserungsbedarfe seitens der Organisationen insgesamt sehr ähnlich ein (Tabelle 20-9 im Online-Anhang). Frauen sehen insgesamt etwas weniger Verbesserungsbedarf bezüglich der einzelnen Aspekte, mit einer Ausnahme: Sie wünschen sich vergleichsweise häufiger Verbesserungen als Männer bei der Anerkennung der Tätigkeit in Form von Zeugnissen, Ausweisen oder Ähnlichem (31,5 Prozent der engagierten Frauen und 28,6 Prozent der engagierten Männer). Möglicherweise spiegelt sich in diesem Ergebnis auch wider, dass Frauen und
Männer zum Teil unterschiedliche freiwillige Tätigkeiten übernehmen. Freiwillig engagierte Männer haben zum Beispiel häufiger eine Leitungsfunktion, mit der möglicherweise auch eine höhere Sichtbarkeit und Anerkennung der Leistung durch andere einhergeht (siehe Kapitel 11).

Zudem wird die Frage der Anerkennung von Engagierten aus den jüngeren und älteren Altersgruppen unterschiedlich eingeschätzt (Tabelle 20-9 im Online-Anhang): Bei der Anerkennung ihrer freiwilligen Tätigkeit in Form von Zeugnissen, Ausweisen oder Ähnlichem sehen 40,2 
Prozent der 14- bis 29-Jährigen Verbesserungsbedarf, aber lediglich 19,2 Prozent der 65 Jahre und älteren Engagierten. Dies ist insofern nachvollziehbar, als sich Jüngere hier möglicherweise Vorteile auch für ihren weiteren Lebensweg erhoffen - etwa auf dem Arbeitsmarkt, auf dem der Zugang zu erstrebenswerten beruflichen Positionen in starkem Maße durch das Vorweisen beglaubigter Zeugnisse und Zertifikate geregelt ist -, während die berufliche Verwertbarkeit von Zeugnissen für ältere Engagierte keine entscheidende Rolle mehr spielt. Das bedeutet aber nicht, dass sich ältere Engagierte keine Anerkennung wünschen, sondern lediglich, dass die Anerkennung eher in anderer Form erwünscht zu sein scheint.

Betrachten wir die Ergebnisse bezüglich des Verbesserungsbedarfs bei der Anerkennung der Tätigkeit durch hauptamtliche Kräfte in der Organisation, sehen ältere Engagierte diesen Bedarf tatsächlich sogar anteilig häufiger als jüngere Engagierte: 31,7 Prozent der 14- bis 29-jährigen Engagierten, aber 38,5 Prozent der 65-jährigen und älteren Engagierten bejahen, dass dieser Bedarf besteht. Die Wertschätzung durch hauptamtliche Kräfte der Organisation scheint sich für die älteren Engagierten noch wichtiger darzustellen als für die jüngeren. Die 65-jährigen und älteren Engagierten nennen darüber hinaus am seltensten Verbesserungsbedarf bezüglich der finanziellen Vergütung ihrer Arbeit (lediglich 16,8 Prozent). Auch diese Form der Anerkennung scheint somit viel weniger bedeutsam als die persönliche Anerkennung durch die Hauptamtlichen in den Organisationen. Ansonsten stimmen die Engagierten in verschiedenen Altersgruppen bezüglich der Reihenfolge der Wichtigkeit verschiedener Verbesserungsbedarfe in den Organisationen weitgehend überein.

Insgesamt schätzen niedrig und hochgebildete Engagierte die Verbesserungsbedarfe sehr ähnlich ein. Bei den meisten Themen wie der Bereitstellung geeigneter Räume sind die Bildungs- unterschiede vergleichsweise gering, mit zwei Ausnahmen: Freiwillig Engagierte mit niedriger Bildung wünschen sich deutlich häufiger Verbesserungen bei der Anerkennung der Tätigkeit durch hauptamtliche Kräfte und bei der finanziellen Anerkennung (in Form einer Vergütung für die geleistete Arbeit) als freiwillig Engagierte mit hoher Bildung (Tabelle 20-10 im Online-Anhang). Die Gruppe der freiwillig engagierten Schülerinnen und Schüler hebt sich vor allem dadurch ab, dass sie deutlich seltener Verbesserungsbedarfe nennt. Insbesondere bei einer unbürokratischen Kostenerstattung ist dies plausibel, vor dem Hintergrund, dass den Schülerinnen und Schülern von allen Engagierten am seltensten Kosten in ihrem Engagement entstehen (siehe Kapitel 14). Lediglich bei der Anerkennung ihrer freiwilligen Tätigkeit in Form von Zeugnissen, Ausweisen oder Ähnlichem sehen Schülerinnen und Schüler häufiger Verbesserungsbedarfe als die anderen Engagierten. Dies ist wie bei den jüngeren Engagierten insgesamt vor allem vor dem Hintergrund der Hoffnung nachvollziehbar, dass sich dadurch auch ihre künftigen Chancen auf dem Arbeitsmarkt verbessern würden, etwa einen Ausbildungs- oder Arbeitsplatz zu finden oder eine bessere Bezahlung zu erhalten.

In unterschiedlichen Organisationsformen sehen die Engagierten unterschiedliche Verbesserungsbedarfe (Abbildung 20-8): Engagierte in individuell organisierten Gruppen geben häufiger Räume und Ausstattungsmittel als Verbesserungsbedarfe an als Engagierte in allen anderen Organisationsformen, was sicherlich damit $\mathrm{zu}$ tun hat, dass in den formalen Organisationen mehr und vielleicht auch bessere Immobilien genutzt werden können. Besonders bei den Kirchen und religiösen Vereinigungen werden hingegen am wenigsten Verbesserungsbedarfe gesehen, mit einer Ausnahme: bei der Anerkennung der Tätigkeit durch hauptamtliche Kräfte in der Organisation. 


\section{Abbildung 20-8: Verbesserungsmöglichkeiten durch die Organisationen 2014, nach Organisationsform}

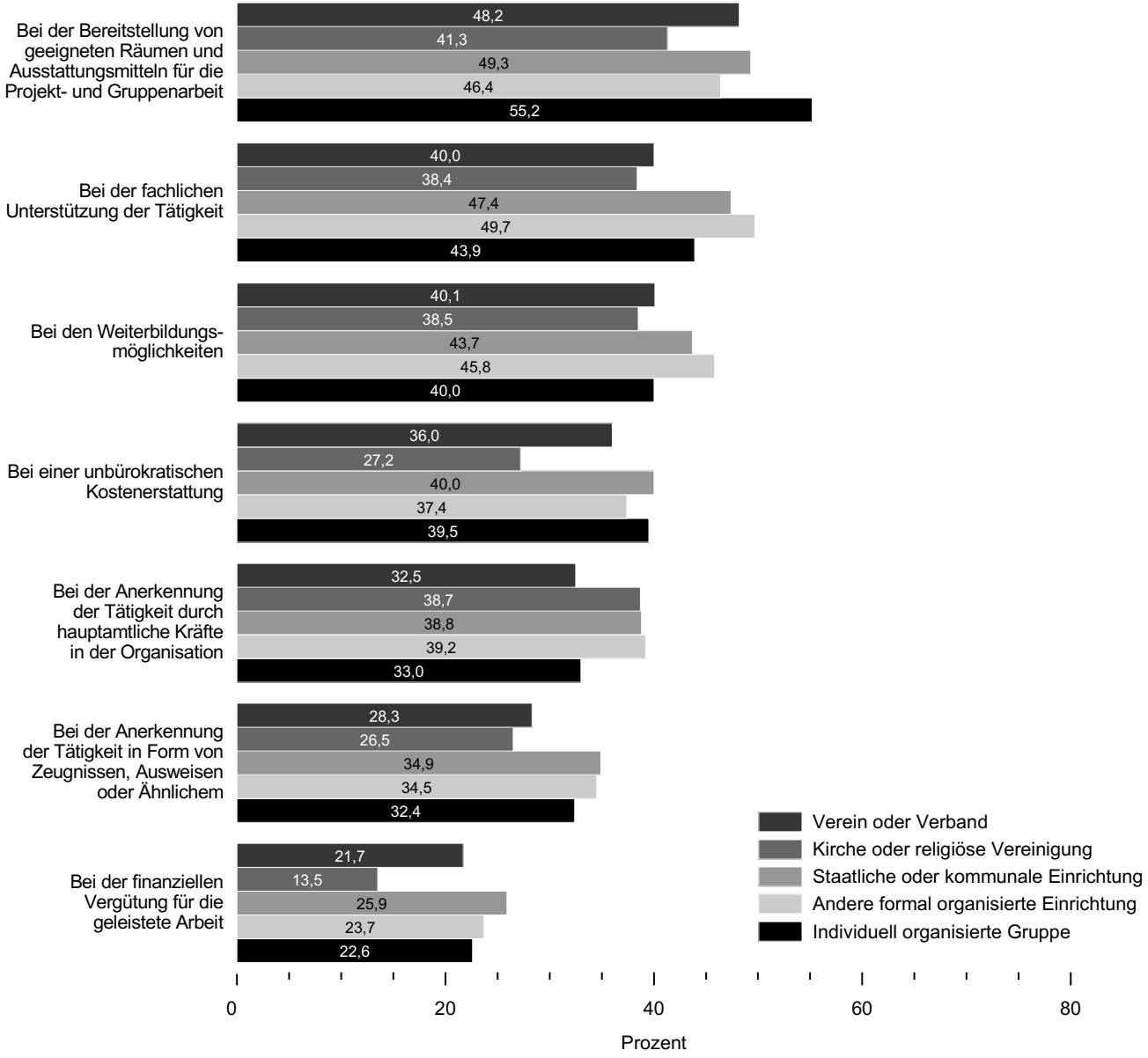

Quelle: FWS 2014, gewichtet, eigene Berechnungen (DZA). Basis: Alle Engagierten. Mehrfachnennungen möglich. Bereitstellung von Räumen $(n=12.343)$, Fachliche Unterstützung $(n=12.339)$, Weiterbildung $(n=12.318)$, Kostenerstattung $(n=12.240)$, Anerkennung durch Hauptamtliche $(n=12.253)$, Anerkennung durch Zeugnisse $(n=12.327)$, Vergütung $(n=12.315)$. 


\subsubsection{Verbesserungsmöglichkeiten durch Staat und Gesellschaft}

Die Engagierten sehen zu hohen Anteilen Verbesserungsmöglichkeiten in Bezug auf ihre Organisation, aber noch zu deutlich höheren Anteilen bei den Rahmenbedingungen bezüglich des Staates und der Gesellschaft. Aus Sicht der Engagierten sollte von staatlicher und gesell- schaftlicher Seite mehr über Gelegenheiten zum ehrenamtlichen oder freiwilligen Engagement informiert und beraten werden, eine Mehrheit von 58,8 Prozent der Engagierten stimmt dem zu (Abbildung 20-9). Auch wünschen sich mehr als die Hälfte der Engagierten Verbesserungen bei der Absicherung durch Haftpflicht- und Unfallversicherung.

\section{Abbildung 20-9: Verbesserungsmöglichkeiten durch Staat und Gesellschaft 2014}

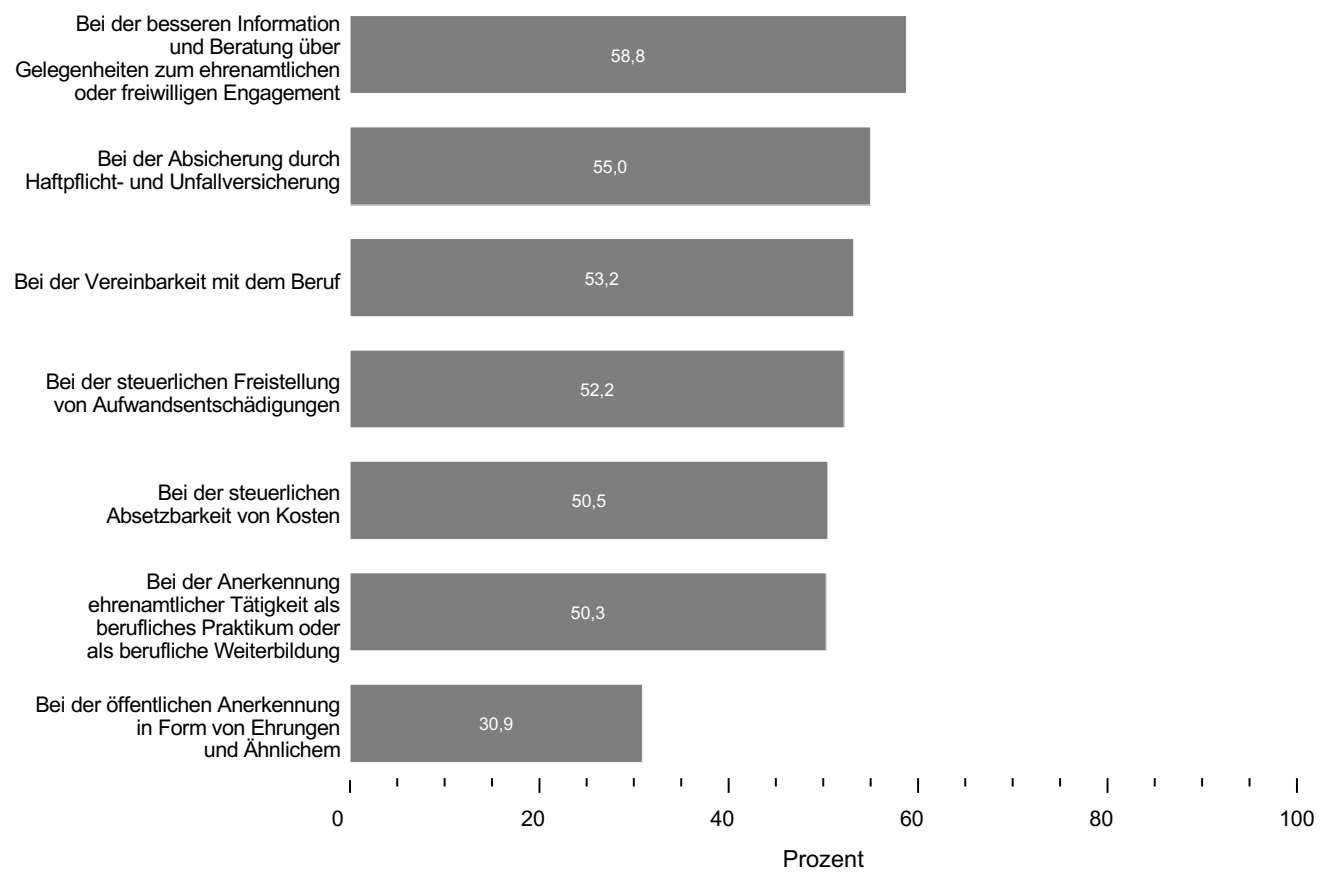

Quelle: FWS 2014, gewichtet, eigene Berechnungen (DZA). Basis: Alle Engagierten, Mehrfachnennungen möglich. Information und Beratung $(n=12.282)$, Versicherung $(n=12.080)$, Vereinbarkeit $(n=12.187)$, Steuerliche Freistellung $(n=12.027)$, Steuerliche Absetzbarkeit $(n=12.119)$, Anerkennung als Praktikum $(n=12.204)$, Anerkennung durch Ehrungen $(n=12.326)$.

Hinsichtlich der Anerkennung ihres Engagements sieht rund die Hälfte aller Engagierten Verbesserungsbedarf bei der Anerkennung ehrenamtlicher Tätigkeiten als berufliches Praktikum oder als berufliche Weiterbildung. Lediglich ein knappes Drittel der Engagierten wünscht sich Verbesserungen bei der öffentlichen Aner- kennung in Form von Ehrungen und Anderem. Hier scheint tatsächlich die Verwertbarkeit des Nachweises über eine ehrenamtliche Tätigkeit etwa im Studium oder auf dem Arbeitsmarkt im Vordergrund zu stehen.

Wie in den Organisationen sehen Frauen auch auf Seiten des Staates und der Gesellschaft 
zu einem höheren Anteil als Männer einen Verbesserungsbedarf bei der Anerkennung (Tabelle 20-12 im Online-Anhang). Bei der Anerkennung ehrenamtlicher Tätigkeit als berufliches Praktikum oder als berufliche Weiterbildung sehen engagierte Frauen sogar (54,8 Prozent) anteilig deutlich häufiger Verbesserungsmöglichkeiten als engagierte Männer (46,0 Prozent). Eine Ausnahme ist die öffentliche Anerkennung des freiwilligen Engagements in Form von Ehrungen. Hier äußern 33,9 Prozent der Männer und 27,7 Prozent der Frauen Verbesserungsbedarf.

Parallel zum Verbesserungsbedarf seitens der Organisationen zeigen sich auch beim Verbesserungsbedarf auf der Seite von Staat und Gesellschaft Altersgruppenunterschiede bezüglich verschiedener Formen der Anerkennung (Tabelle 20-12 im Online-Anhang). Die Anerkennung ehrenamtlicher Tätigkeit als berufliches Praktikum oder als berufliche Weiterbildung nennen 53,7 Prozent der 14- bis 29-Jährigen, aber nur 40,1 Prozent der 65-jährigen und älteren Engagierten. Mehr öffentliche Anerkennung in Form von Ehrungen und Ähnlichem nennen hingegen 31,6 Prozent der 14- bis 29-jährigen Engagierten und 33,2 Prozent der 65-jährigen und älteren Engagierten. Bei der ältesten Altersgruppe ist der Wunsch nach öffentlicher Anerkennung möglicherweise deshalb wichtiger als bei den anderen Gruppen, weil Alternativen wie die Anerkennung beruflicher Leistungen durch den Eintritt in den Ruhestand wegfallen.

Verbesserungen bei der Vereinbarkeit von freiwilligem Engagement mit dem Beruf nennen mit 53,2 Prozent ebenfalls mehr als die Hälfte aller Engagierten, und hier unterscheiden sich die Anteile von engagierten Frauen und engagierten Männer nicht. Nach Altersgruppen sehen wir allerdings, dass insbesondere die 30- bis 49-Jäh- rigen mit 59,4 Prozent hier Handlungsbedarf sehen, was insofern plausibel erscheint, dass in dieser Lebensphase die Wahrscheinlichkeit, selbst minderjährige Kinder zu versorgen, am größten ist. Darüber hinaus können auch die Arbeitgeberinnen und Arbeitgeber der Engagierten manches tun, um das Engagement ihrer Mitarbeiterinnen und Mitarbeiter zu unterstützen.

Bei den Verbesserungsbedarfen durch Staat und Gesellschaft zeigt sich, dass sich engagierte Schülerinnen und Schüler in einer spezifischen Lebenssituation befinden, die noch nicht durch den Erwerbsarbeitsdruck und finanzielle Erwägungen gekennzeichnet ist (Tabelle 20-13 im Online-Anhang). So sehen sie deutlich seltener Verbesserungsbedarf bei der Vereinbarkeit des Engagements mit dem Beruf und bei den steuerlichen Aspekten (Freistellung von Aufwandsentschädigungen und Absetzbarkeit von Kosten). Für Engagierte mit hoher Bildung sind die genannten Aspekte jedoch wichtiger als für Engagierte mit niedriger Bildung, die zudem zu größeren Teilen nicht erwerbstätig sind. Dies ist auch darauf zurückzuführen, dass Personen höheren Alters, die sich bereits im Ruhestand befinden, zu größeren Anteilen lediglich einen niedrigen Bildungsabschluss haben. Die Vereinbarkeit mit dem Beruf sowie steuerliche Aspekte spielen für sie daher keine oder zumindest eine nachrangige Rolle. Insgesamt nennen Engagierte sowohl mit niedriger Bildung als auch mit hoher Bildung jedoch ähnliche Verbesserungsbedarfe, hier zeigen sich nur graduelle Unterschiede. Engagierte in Vereinen und Verbänden und im Besonderen die Engagierten in Kirchen und religiösen Vereinigungen sehen insgesamt weniger Verbesserungsbedarfe als Engagierte in anderen Organisationsformen (Abbildung 20-10). 
Abbildung 20-10: Verbesserungsmöglichkeiten durch Staat und Gesellschaft 2014, nach Organisationsform

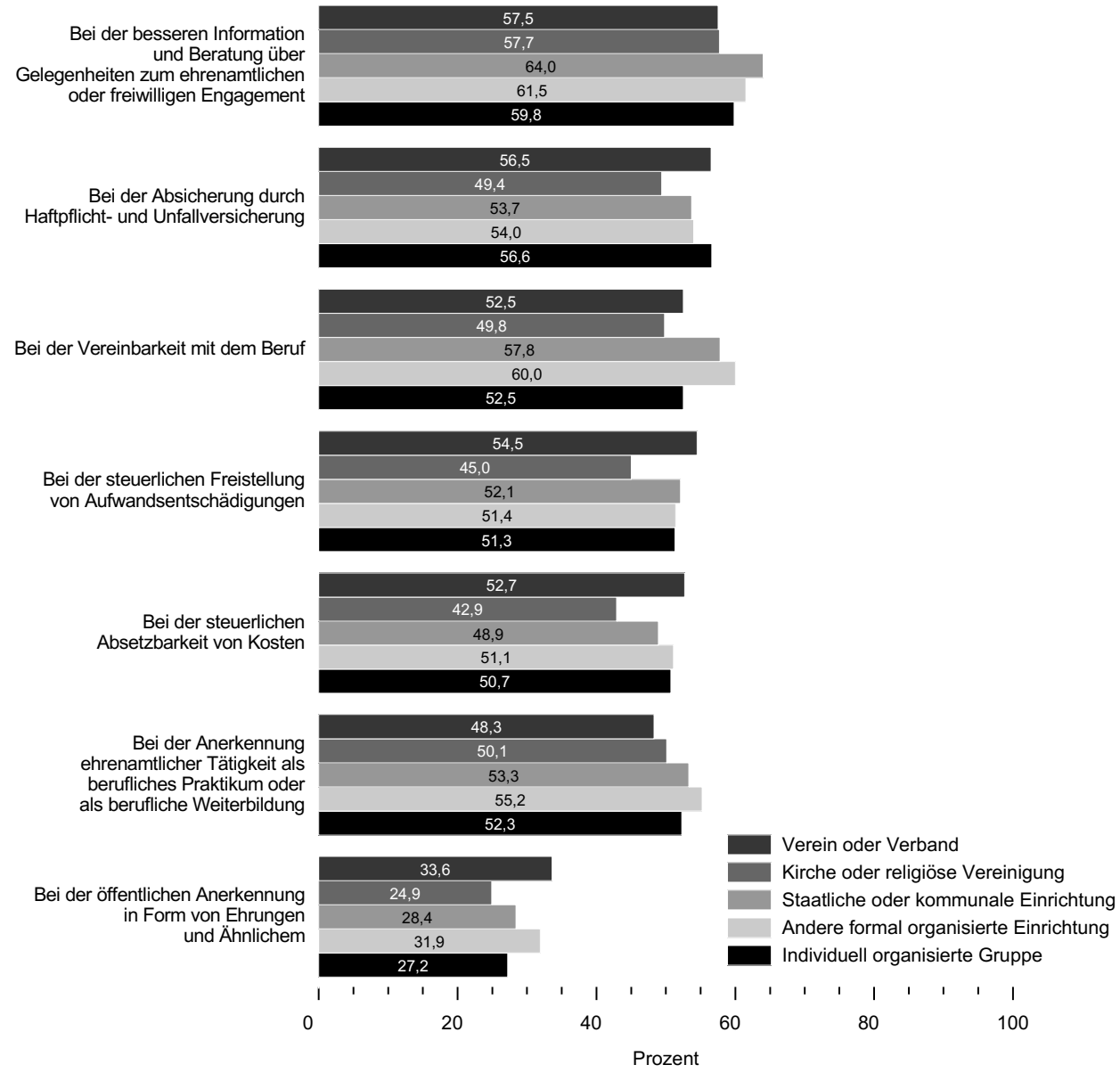

Quelle: FWS 2014, gewichtet, eigene Berechnungen (DZA). Basis: Alle Engagierten, Mehrfachnennungen möglich. Information und Beratung $(n=12.282)$, Versicherung $(n=12.080)$, Vereinbarkeit $(n=12.187)$, Steuerliche Freistellung $(n=12.027)$, Steuerliche Absetzbarkeit $(n=12.119)$, Anerkennung als Praktikum $(n=12.204)$, Anerkennung durch Ehrungen $(n=12.326)$.

\subsubsection{Unterstützung durch den Arbeitgeber}

Auch Unternehmen können die Rahmenbedingungen für freiwilliges Engagement verbessern, indem sie es ihren Beschäftigten erleichtern, ihr freiwilliges Engagement mit ihrer beruflichen Tätigkeit zu vereinbaren. Sicherlich benötigen nicht alle erwerbstätigen Engagierten Unterstützung, für manche freiwillige Tätigkeiten ist diese jedoch unerlässlich, etwa wenn sie wie die Ausübung eines Schöffenamtes oder einer Tätigkeit im Zivilund Katastrophenschutz in die Arbeitszeit fallen und eine Freistellung dafür notwendig ist.

Insgesamt erhält ein gutes Drittel aller freiwillig Engagierten, die abhängig beschäftigt sind, eine solche Unterstützung durch den Arbeitgeber (Abbildung 20-11a). Von den freiwillig engagier- 
ten Männern ist es mit 37,8 Prozent ein etwas höherer Anteil als von den Frauen mit 33,6 Prozent. Darüber hinaus erhalten die Engagierten in der jüngsten Altersgruppe der 14- bis 29-Jährigen, die bereits abhängig beschäftigt sind, anteilig am häufigsten Unterstützung durch den Arbeitgeber (44,1 Prozent), und ebenso Engagierte mit niedriger Bildung (38,6 Prozent).

\section{Abbildung 20-11: Unterstützung durch den Arbeitgeber 2014, a) gesamt, nach Geschlecht sowie nach Alter und b) nach Bildung}

a) gesamt, nach Geschlecht sowie nach Alter

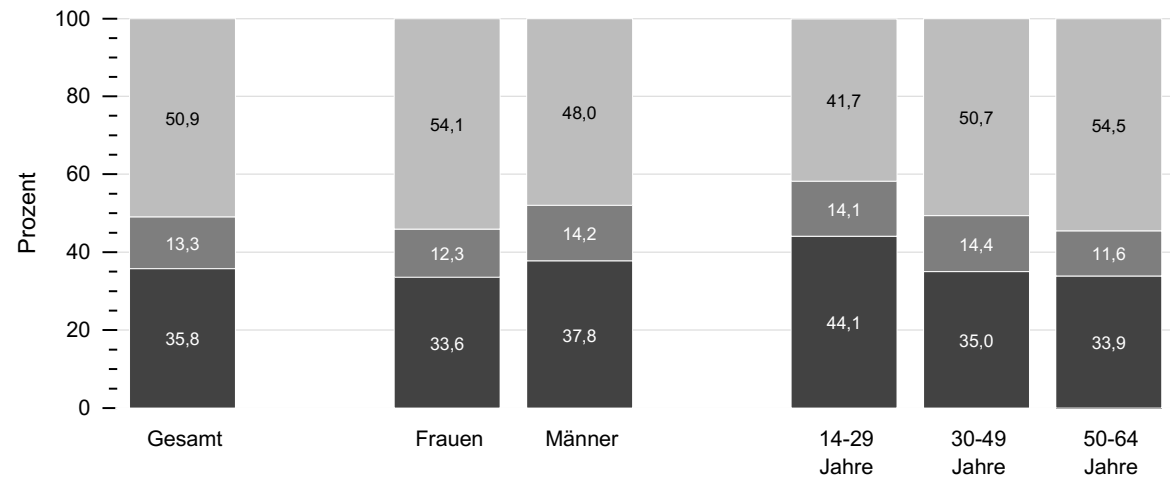

\section{b) nach Bildung}

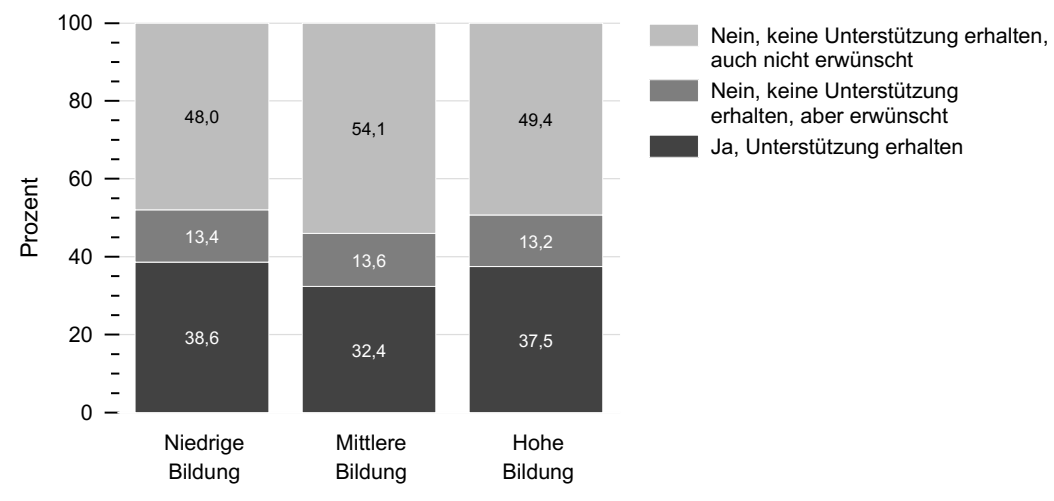

Quelle: FWS 2014, gewichtet, eigene Berechnungen (DZA). Basis: alle Engagierten, die abhängig beschäftigt sind, $(n=5.437)$. Die Gruppe 65 Jahre und älter wird aufgrund geringer Fallzahlen abhängig Beschäftigter nicht dargestellt. Die Gruppe der Schülerinnen und Schüler wird nicht dargestellt, da sich die Frage nur an abhängig Beschäftigte richtete.

Gleichzeitig wünschen sich 14,2 Prozent der engagierten Männer Unterstützung, erhalten diese aber nicht. Der vergleichbare Wert bei den engagierten Frauen liegt ähnlich hoch (12,3 Prozent). Für diese relativ kleine Gruppe aus dem Kreise aller freiwillig engagierter Arbeitnehmerinnen und Arbeitnehmer könnte ein entsprechender Einsatz ihres Arbeitgebers eine deutliche Verbesserung bringen. Die jeweils größte Gruppe der abhängig beschäftigten und engagierten 
Frauen und Männer erhält jedoch keine Unterstützung durch den Arbeitgeber, wünscht sich aber auch eine solche nicht; bei letzteren scheint kein Handlungsbedarf zu bestehen. Was wir al- lerdings auf Basis dieser Befunde nicht ausschließen können ist, dass sich Personen erst gar nicht freiwillig engagieren, weil sich dies nicht mit ihrer beruflichen Tätigkeit vereinbaren lässt.

\subsection{Fazit}

Hinsichtlich des organisatorischen Rahmens ist ein Trend festzustellen, dass freiwilliges Engagement außerhalb von Vereinen und Verbänden an Bedeutung gewinnt. Insbesondere Frauen engagieren sich im Zeitvergleich anteilig häufiger außerhalb von Vereinen und Verbänden. Insgesamt ist eine steigende Tendenz vor allem beim Engagement in individuell organisierten Gruppen zu verzeichnen.

Knapp zwei Drittel aller Engagierten sind in einer Organisation freiwillig tätig, in der es einen Ansprechpartner oder eine Ansprechpartnerin für sie gibt. Engagierte in individuell organisierten Gruppen geben allerdings anteilig seltener an, dass Ansprechpartnerinnen oder Ansprechpartner vorhanden sind. Dies ist möglicherweise auf einen generell geringeren Formalisierungsgrad als etwa bei kirchlichen Organisationen zurückzuführen. In diesen sowie in staatlichen oder kommunalen Einrichtungen gibt es eher solche Ansprechpersonen als in anderen Organisationsformen.

Bezüglich der organisationalen Rahmenbedingungen zeigt sich auch, dass die Engagierten unterschiedliche Möglichkeiten zur Mitsprache in ihren Organisationen haben: Insgesamt bewerten mehr als drei Viertel aller Engagierten ihre Mitsprachemöglichkeiten als sehr gut beziehungsweise eher gut; Engagierte in individuell organisierten Gruppen bewerten die Mitsprachemöglichkeiten am häufigsten als sehr gut beziehungsweise eher gut (83,8 Prozent). Auch die Mitsprachemöglichkeiten in Vereinen und Verbänden werden überdurchschnittlich oft als sehr gut beziehungsweise eher gut bewertet (78,8 Prozent).

Trotz der überwiegend als gut beziehungsweise sehr gut bewerteten Mitsprachemöglichkeiten sehen die Engagierten zum Teil Verbesserungsbedarfe bei den Organisationen, am häufigsten bei der Bereitstellung geeigneter Räume und Ausstattungsmittel für die Projekt- und Gruppenarbeit. Eine wichtige Unterstützung freiwilligen Engagements ist also zunächst einmal die Bereitstellung ausreichender Mittel.

Aus Sicht der Engagierten könnte ein Hindernis für das freiwillige Engagement in der Doppeloder Dreifachbelastung durch Beruf, Familie und Engagement beziehungsweise informelle Unterstützungsleistungen im sozialen Nahraum bestehen (siehe Kapitel 9). Deshalb sollten gleichzeitig die Rahmenbedingungen für die Vereinbarkeit der unterschiedlichen Aufgaben in diesen Lebensbereichen verbessert werden. Aufgrund der stärkeren Eingebundenheit von Frauen in familiäre und haushaltsbezogene Aufgaben könnte man erwarten, dass insbesondere Frauen eine Vereinbarkeitsproblematik benennen. In der Tat sehen mehr als die Hälfte der Engagierten Verbesserungsmöglichkeiten bei der Vereinbarkeit von freiwilligem Engagement mit dem Beruf. Dieser Bedarf wird aber nicht nur von Frauen, sondern gleichermaßen auch von Männern benannt. Insbesondere für die Altersgruppe der 30- bis 49-Jährigen ist dieser Punkt wichtig, denn in dieser Altersgruppe gibt es besonders viele Personen, die sich in der Familienphase befinden und sowohl privat als auch beruflich mit hohen Anforderungen konfrontiert sind. Zudem engagiert sich in dieser Altersgruppe ein besonders hoher Anteil von Personen (siehe Kapitel 3).

Für die jüngeren Engagierten ist zudem die Anerkennung ihres freiwilligen Engagements in Form von Zeugnissen wichtiger als für andere Altersgruppen, nicht aber in Form einer finan- 
ziellen Vergütung. Um die Anerkennungskultur des freiwilligen Engagements tatsächlich zu stärken, erscheint es daher nicht sinnvoll, auf eine direkte monetäre Vergütung des Engagements $\mathrm{zu}$ setzen. Vielversprechend ist es jedoch, Anerkennung durch Qualifizierung zu ermöglichen, sei es durch die Bescheinigung des Engagements in Form von Zeugnissen oder aber dadurch, dass die Engagierten in ihrer freiwilligen Tätigkeit weitergebildet werden.
Den größten Verbesserungsbedarf sehen alle Altersgruppen bei der Information und Beratung über Gelegenheiten zum ehrenamtlichen oder freiwilligen Engagement. Um mehr Menschen für freiwilliges Engagement zu mobilisieren und es ihnen zu ermöglichen, eine passende freiwillige Tätigkeit zu finden, wäre es daher sinnvoll, Informations- und Beratungsangebote weiter auszubauen.

\section{Literatur}

Alscher, M. (2010). Auf der Suche nach Balance. Frauen und Männer zwischen Beruf, Familie und Engagement. WZB Mitteilungen, 2010(129), 31-33.

Alscher, M., Droß, P. J., Priller, E., \& Schmeißer, C. (2013). Vereine an den Grenzen der Belastbarkeit. WZBrief Zivilengagement, 7, 2-7.

Alscher, M., \& Priller, E. (2011). Organisationsbezogene Daten. In: T. Olk \& B. Hartnuß (Hrsg.) Handbuch bürgerschaftliches Engagement (S. 719-731). Weinheim: Beltz Juventa.

Backes, G. M. (2011). Geschlechterdifferenz im Engagement. In: T. Olk \& B. Hartnuß (Hrsg.) Handbuch Bürgerschaftliches Engagement (S. 65-75). Weinheim: Beltz Juventa.

Coenen-Marx, C. (2011). Ehrenamtliches Engagement in der Kirche. In: T. Olk \& B. Hartnuß (Hrsg.) Handbuch Bürgerschaftliches Engagement (S. 257-265). Weinheim: Beltz Juventa.

Enquete-Kommission ,Zukunft des Bürgerschaftlichen Engagements' (2002). Bericht der Enquete-Kommission ,Zukunft des Bürgerschaftlichen Engagements'. Bürgerschaftliches Engagement: auf dem Weg in eine zukunftsfähige Bürgergesellschaft (Bundestagsdrucksache 14/8900). Berlin: Deutscher Bundestag.

Gensicke, T. (2015). Freiwilliges Engagement in Deutschland: Freiwilligensurvey 2009. Wiesbaden: Springer VS.

Grunow, D. (2011). Selbsthilfe. In: T. Olk \& B. Hartnuß (Hrsg.) Handbuch Bürgerschaftliches Engagement (S. 173-183). Weinheim: Beltz Juventa.

Heinze, R. G. (2011). Verbände. In: T. Olk \& B. Hartnuß (Hrsg.) Handbuch Bürgerschaftliches Engagement (S. 465-473). Weinheim: Beltz Juventa.

Köhler, H. (2015). Bürgerliches Gesetzbuch BGB (76. Aufl.). München: Deutscher Taschenbuch Verlag.

Krimmer, H., \& Priemer, J. (2013). ZIVIZ-Survey 2012. Zivilgesellschaft verstehen. Berlin: Stifterverband für die Deutsche Wissenschaft.

Reiser, M. (2011). Kommunalpolitisches Ehrenamt. In: T. Olk \& B. Hartnuß (Hrsg.) Handbuch Bürgerschaftliches Engagement (S. 291-303). Weinheim: Beltz Juventa.

Sachße, C. (2011). Traditionslinien bürgerschaftlichen Engagements in Deutschland. In: T. Olk \& B. Hartnuß (Hrsg.) Handbuch Bürgerschaftliches Engagement (S. 17-27). Weinheim: Beltz Juventa.

Salamon, L. M., \& Sokolowski, W. S. ( 2003). Institutional Roots of Volunteering. In: P. Dekker \& L. Halmann (Hrsg.) The Values of Volunteering: Cross-Cultural Perspectives (S. 71-90). New York: Kluwer Academic.

Schumacher, J. (2015). Kooperation von Haupt- und Ehrenamtlichen in Pflege, Sport und Kultur. Endbericht. Berlin: Bundesministerium für Familie, Senioren, Frauen und Jugend.

Weßels, B. (2013). Politische Integration und politisches Engagement. In: Statistisches Bundesamt \& Wissenschaftszentrum Berlin für Sozialforschung (Hrsg.) Datenreport 2013. Ein Sozialbericht für die Bundesrepublik Deutschland (S. 363-376). Bonn: Bundeszentrale für politische Bildung. 
Zeman, P. (2012). Selbsthilfe - Organisationen und Formen. In: H.-W. Wahl, C. Tesch-Römer \& J. P. Ziegelmann (Hrsg.) Angewandte Gerontologie. Interventionen für ein gutes Altern in 100 Schlüsselbegriffen (S. 541-546). Stuttgart: Kohlhammer.

Zimmer, A. (2011). Vereine. In: T. Olk \& B. Hartnuß (Hrsg.) Handbuch Bürgerschaftliches Engagement (S. 453-463). Weinheim: Beltz Juventa.

Open Access Dieses Kapitel wird unter der Creative Commons Namensnennung 4.0 International Lizenz (http://creativecommons.org/licenses/by/4.0/deed.de) veröffentlicht, welche die Nutzung, Vervielfältigung, Bearbeitung, Verbreitung und Wiedergabe in jeglichem Medium und Format erlaubt, sofern Sie den/die ursprünglichen Autor(en) und die Quelle ordnungsgemäß nennen, einen Link zur Creative Commons Lizenz beifügen und angeben, ob Änderungen vorgenommen wurden.

Die in diesem Kapitel enthaltenen Bilder und sonstiges Drittmaterial unterliegen ebenfalls der genannten Creative Commons Lizenz, sofern sich aus der Abbildungslegende nichts anderes ergibt. Sofern das betreffende Material nicht unter der genannten Creative Commons Lizenz steht und die betreffende Handlung nicht nach gesetzlichen Vorschriften erlaubt ist, ist für die oben aufgeführten Weiterverwendungen des Materials die Einwilligung des jeweiligen Rechteinhabers einzuholen. 